\title{
SOLUTIONS CONTAINING A LARGE PARAMETER OF A QUASI-LINEAR HYPERBOLIC SYSTEM OF EQUATIONS AND THEIR NONLINEAR GEOMETRIC OPTICS APPROXIMATION
}

\author{
ATSUSHI YOSHIKAWA
}

\begin{abstract}
It is well known that a quasi-linear first order strictly hyperbolic system of partial differential equations admits a formal approximate solution with the initial data $\lambda^{-1} a_{0}(\lambda x \cdot \eta, x) r_{1}(\eta), \lambda>0, x, \eta \in \mathbf{R}^{n}, \eta \neq 0$. Here $r_{1}(\eta)$ is a characteristic vector, and $a_{0}(\sigma, x)$ is a smooth scalar function of compact support. Under the additional requirements that $n=2$ or 3 and that $a_{0}(\sigma, x)$ have the vanishing mean with respect to $\sigma$, it is shown that a genuine solution exists in a time interval independent of $\lambda$, and that the formal solution is asymptotic to the genuine solution as $\lambda \rightarrow \infty$.
\end{abstract}

\section{INTRODUCTION}

Consider the following quasi-linear system of partial differential equations of first order:

$$
A_{0}(u) \frac{\partial}{\partial t} u+\sum_{j=1}^{n} A_{j}(u) \frac{\partial}{\partial x_{j}} u+B(u) u=0,
$$

$x \in \mathbf{R}^{n}, t \geq 0$. Here $u=u(x, t)$ is an $m$-vector-valued unknown function, which is required to satisfy the initial condition

$$
u(x, 0)=g(x), \quad x \in \mathbf{R}^{n},
$$

$g(x)$ being a given $m$-vector-valued function. In passing, we remind the reader that all the vectors, matrices or functions in the present paper are real valued unless the contrary is explicitly mentioned.

We suppose the system $(0.1)$ is positive symmetric and strictly hyperbolic. More precisely, all its coefficient matrices $A_{0}(u), \ldots, A_{n}(u), B(u)$ are supposed to be symmetric except $B(u)$ while $A_{0}(u)$ to be positive definite. All their entries are supposed to be smooth in $u \in \mathbf{R}^{m}$. Furthermore, as the system being strictly hyperbolic, the characteristic equation

$$
\operatorname{det}\left(-\tau A_{0}(u)+\sum_{j=1}^{n} \xi_{j} A_{j}(u)\right)=0
$$

Received by the editors January 20, 1991.

1991 Mathematics Subject Classification. Primary 35L60; Secondary 41A60, 41A63.

This work is supported in part by Grant-in-Aid for Scientific Research, Ministry of Education, Science and Culture, Japanese Government No. 62460005. 
is assumed to have $m$ distinct real roots $\tau=p_{1}(u, \xi), \ldots, \tau=p_{m}(u, \xi)$ for each $u \in \mathbf{R}^{m}, \xi=\left(\xi_{1}, \ldots, \xi_{n}\right) \in \mathbf{R}^{n} \backslash 0$. We require that $p_{1}(u, \xi), \ldots$, $p_{m}(u, \xi)$ be smooth in $u$ and $\xi$ in a neighborhood of $u=0$ and $\xi=\eta$, where $\eta$ is a given nonzero element of $\mathbf{R}^{n}$. We also stipulate that at least one, say the first, characteristic field is genuinely nonlinear, or the scalar product

$$
\nabla_{u} p_{1}(u, \xi) \cdot r_{1}(u, \xi) \neq 0
$$

in a neighborhood of $u=0$ and $\xi=\eta$. Here $r_{1}(u, \xi)$ is an eigenvector corresponding to the eigenvalue $p_{1}(u, \xi)$ :

$$
p_{1}(u, \xi) A_{0}(u) r_{1}(u, \xi)=\sum_{j=1}^{n} \xi_{j} A_{j}(u) r_{1}(u, \xi) .
$$

The detailed requirements on the system $(0.1)$ will be given in $\S 1$.

Let $n \geq 2$. Suppose $g=g(x)$ is regular enough that $g$ belongs to the Sobolev space over $\mathbf{R}^{n}$ of order $s>n / 2+1$. Then it is well known that the initial value problem $(0.1),(0.2)$ admits a unique "regular" solution $u(x, t)$, which is valid in a certain time interval $0 \leq t \leq T_{g}$. Here $T_{g}$ depends on the size of the Sobolev norm $\|g\|_{s}$. Generally speaking, $T_{g} \geq O\left(1 /\|g\|_{s}\right)$ (see Kato [5, 6], Majda [9], Klainerman [7]).

Now let us take as the initial data an $m$-vector-valued function which depends on a parameter $\lambda \geq \lambda_{0}>0$. Let

$$
g(x)=g(x, \lambda)=\lambda^{-1} a_{0}(\lambda x \cdot \eta, x) r_{1}(0, \eta) .
$$

Here $\eta \in \mathbf{R}^{n}, \eta \neq 0$, and $x \cdot \eta$ is the inner product of $\mathbf{R}^{n}: x \cdot \eta=\sum_{1}^{n} x_{j} \eta_{j}$, $x=\left(x_{1}, \ldots, x_{n}\right), \eta=\left(\eta_{1}, \ldots, \eta_{n}\right), a_{0}(\sigma, x)$ is a smooth scalar function of $(\sigma, x) \in \mathbf{R} \times \mathbf{R}^{n} . r_{1}(0, \eta)$ is the eigenvector mentioned in the above. Recall that an initial data of the form $\exp \{\lambda \sqrt{-1} x \cdot \eta\} u_{0}(x)$ is the basic ingredient of the geometric optics approach to linear hyperbolic partial differential equations. Recall also that, in our nonlinear situation, if $a_{0}(\sigma, x)$ is "nice", for instance, is compactly supported, then a formal asymptotic solution, say $U(x, t, \lambda)$, of the initial value problem $(0.1),(0.2),(0.5)$ can readily be constructed, so that it is smooth in a time interval independent of $\lambda \geq \lambda_{0}$ (see Choquet-Bruhat [1], Hunter and Keller [3], Hunter, Majda, and Rosales [4], Majda [10], and also our discussions in $\S 2$ ).

On the other hand, even if $a_{0}(\sigma, x)$ is compactly supported, it can easily be seen that the Sobolev norms of $g(x, \lambda)$ behave as

$$
\|g(\cdot, \lambda)\|_{s} \leq C_{s} \lambda^{s-1}
$$

for $\lambda \geq \lambda_{0}$ and $s \geq 0$. Therefore, existence of the solution $u(x, t, \lambda)$ of the initial value problem may only be assessed for time intervals depending on $\lambda$, which moreover shrink to zero as $\lambda$ grows to infinity. It would thus be interesting to know under what condition $u(x, t, \lambda)$ is valid in a time interval independent of $\lambda$ so that $u(x, t, \lambda) \sim U(x, t, \lambda)$ asymptotically as $\lambda \rightarrow \infty$ then.

The purpose of the present paper is to show in low dimensions, i.e., $n=2$ or 3 , this is in fact the case provided $a_{0}(\sigma, x)$ is compactly supported and smooth enough, that is,

$$
a_{0}(\sigma, x) \in C_{0}^{N}\left(\mathbf{R} \times \mathbf{R}^{n}\right), \quad N \text { very large, }
$$


and moreover has the vanishing mean with respect to $\sigma$ :

$$
\int_{\mathbf{R}} a_{0}(\sigma, x) d \sigma=0 \text {. }
$$

Then for some $\lambda_{1}>0$ and for some $T_{1}>0$ independent of $\lambda \geq \lambda_{1}, u(x, t, \lambda)$ and $U(x, t, \lambda)$ are shown to be valid for $0 \leq t \leq T_{1}$ so that $u(x, t, \lambda)=$ $U(x, t, \lambda)+O\left(\lambda^{-3}\right)$ as $\lambda \rightarrow \infty$. Actually, $u(x, t, \lambda)$ and all of its first partial derivatives with respect to $x$ and $t$ are shown to be uniformly bounded for $x \in \mathbf{R}^{n}, 0 \leq t \leq T_{1}, \lambda \geq \lambda_{1}$. It follows then that the solution $u(x, t, \lambda)$ is unique in spite of the estimates $\|u(\cdot, t, \lambda)\|_{s} \leq C_{s} \lambda^{s-1}, 0 \leq s \leq 3$, and $\left\|u_{t}(\cdot, t, \lambda)\right\|_{s} \leq C_{s}^{\prime} \lambda^{s}, 0 \leq s \leq 2$. The precise statements of our results are given in $\S 1$ (see Theorems 1.4 and 1.5). Uniform existence of the solution $u(x, t, \lambda)$ will be proved in $\S 3$.

Remark. Requirements of the form (0.6), (0.7) are closely related to a certain class of functions which generate "wavelets" (see Daubechies [2]).

Further extensions or generalizations of the present results will be discussed elsewhere.

\section{AsSUMPTIONS AND STATEMENT OF THE RESUltS}

Let us begin by making technically precise the assumptions on the system (0.1). Let $w \in \mathbf{R}^{m}$. Denote by $L(w)$ the operator

$$
L(w) v=A_{0}(w) \frac{\partial}{\partial t} v+\sum_{j=1}^{n} A_{j}(w) \frac{\partial}{\partial x_{j}} v+B(w) v
$$

acting on $m$-vector-valued functions $v=v(x, t)$. Since only small $u$ is relevant in the system $(0.1)$, we assume that each of the coefficient matrices $A_{0}(w), A_{1}(w), \ldots, A_{n}(w), B(w)$ of the operator $L(w)$ is decomposed into a sum of an $m \times m$ constant matrix and an $m \times m$ matrix with entries consisting of functions of $w$ belonging to $\mathscr{S}\left(\mathbf{R}^{m}\right)$. Thus, for example,

$$
A_{0}(w)=A_{0}^{c}+A_{0}^{d}(w) \text {, }
$$

where $A_{0}^{c}$ is the constant part and $A_{0}^{d}(w)$ the rapidly decreasing part. Note that such a decomposition is unique. Furthermore, it is easily seen that the matrices admitting this kind of decomposition, to be called decomposable, form a matrix subalgebra, and if a decomposable matrix is invertible, then its inverse is also decomposable.

Returning to the coefficients of $L(w)$, we assume $A_{0}(w), A_{1}(w), \ldots, A_{n}(w)$ are symmetric and $A_{0}(w)$ positive definite as we have mentioned in $\S 0$. Then the inverse $A_{0}(w)^{-1}$ is also positive definite and decomposable. In particular, there are positive constants $\gamma$ and $\Gamma$ independent of $w$ such that

$$
\gamma y \cdot y \leq y \cdot A_{0}(w) y \leq \Gamma y \cdot y
$$

for $y \in \mathbf{R}^{m}, w \in \mathbf{R}^{m}$.

Next recall the characteristic equation $(0.3)$. Let $\eta=\left(\eta_{1}, \ldots, \eta_{n}\right) \in \mathbf{R}^{n}$, $\eta \neq 0$. We assume that the characteristic equation

$$
\operatorname{det}\left(-\tau A_{0}(w)+\sum_{j=1}^{n} \xi_{j} A_{i}(w)\right)=0
$$


has $m$ distinct real roots $\tau=p_{1}(w, \xi), \ldots, p_{m}(w, \xi)$ which are smooth functions of $w \in \mathbf{R}^{m}$ and $\xi \in \mathbf{R}^{n}, \xi \neq 0$, in a neighborhood of $w=0$ and $\xi=\eta$. Thus, $p_{1}(w, \xi), \ldots, p_{m}(w, \xi)$ are real distinct eigenvalues of the matrix

$$
M(w, \xi)=\sum_{j=1}^{n} \xi_{j} \tilde{A}_{j}(w), \quad \widetilde{A}_{j}(w)=A_{0}(w)^{-1} A_{j}(w)
$$

Let $r_{1}(w, \xi), \ldots, r_{m}(w, \xi)$ be right eigenvectors of $M(w, \xi)$ corresponding to $p_{1}(w, \xi), \ldots, p_{m}(w, \xi)$, respectively. Thus,

$$
p_{k}(w, \xi) r_{k}(w, \xi)=M(w, \xi) r_{k}(w, \xi),
$$

$k=1, \ldots, m$. Similarly, we have left eigenvectors $r_{1}^{*}(w, \xi), \ldots, r_{m}^{*}(w, \xi)$ so that

$$
p_{k}(w, \xi) r_{k}^{*}(w, \xi)=r_{k}^{*}(w, \xi) M(w, \xi),
$$

$k=1, \ldots, m$, and moreover in such a way that

$$
r_{k}^{*}(w, \xi) \cdot r_{l}(w, \xi)= \begin{cases}1, & k=l \\ 0, & k \neq l\end{cases}
$$

$k, l=1, \ldots, m$, hold. Then we may suppose $r_{1}(w, \xi), \ldots, r_{m}(w, \xi)$, $r_{1}^{*}(w, \xi), \ldots, r_{m}^{*}(w, \xi)$ are smooth in $w, \xi$ near $w=0$ and $\xi=\eta$.

Remark. In the following, we will omit reference to $w$ when $w=0$ in $p_{k}(w, \xi)$, $r_{k}(w, \xi)$, or $r_{k}^{*}(w, \xi)$. Thus we will simply write $p_{k}(\xi), r_{k}(\xi), r_{k}^{*}(\xi)$ instead of $p_{k}(0, \xi), r_{k}(0, \xi), r_{k}^{*}(0, \xi)$. However, we retain $w=0$ in their Fréchet (-Gâteaux) derivatives with respect to $w$, for instance, $\nabla_{w} p_{k}(0, \xi) \cdot v=$ $\left.(d / d \varepsilon) p_{k}(\varepsilon v, \xi)\right|_{\varepsilon=0}$.

Lemma 1.1. We have

$$
\begin{gathered}
r_{k}^{*}(w, \xi) \cdot \tilde{A}_{j}(w) r_{k}(w, \xi)=p_{k}^{(j)}(w, \xi), \\
r_{l}^{*}(w, \xi) \cdot \tilde{A}_{j}(w) r_{k}(w, \xi) \\
=\left(p_{k}(w, \xi)-p_{l}(w, \xi)\right) r_{l}^{*}(w, \xi) \cdot r_{k}^{(j)}(w, \xi),
\end{gathered}
$$

$k, l=1, \ldots, m, k \neq l ; j=1, \ldots, n$. Here

$$
p_{k}^{(j)}(w, \xi)=\frac{\partial}{\partial \xi_{j}} p_{k}(w, \xi), \quad r_{k}^{(j)}(w, \xi)=\frac{\partial}{\partial \xi_{j}} r_{k}(w, \xi)
$$

Furthermore, for $v \in \mathbf{R}^{m}$,

$$
\begin{aligned}
& r_{k}^{*}(w, \xi) \cdot\left(\nabla_{w} M(w, \xi) \cdot v\right) r_{k}(w, \xi)=\nabla_{w} p_{k}(w, \xi) \cdot v, \\
& \quad r_{l}^{*}(w, \xi) \cdot\left(\nabla_{w} M(w, \xi) \cdot v\right) r_{k}(w, \xi) \\
& \quad=\left(p_{k}(w, \xi)-p_{l}(w, \xi)\right) r_{l}^{*}(w, \xi) \cdot\left(\nabla_{w} r_{k}(w, \xi) \cdot v\right),
\end{aligned}
$$

$k, l=1, \ldots, m, k \neq l$.

In fact, these are consequences of differentiations of (1.5), (1.6), and (1.7) combined with (1.4).

Remarks. 1. Compare (1.10) with (0.4). 
2. If $S_{i j} \in \mathbf{R}$ and $S_{i j}=S_{j i}, i, j=1, \ldots, n$, the following identities hold. They are useful when we consider phase functions of waves which are more general than planar ones. Namely, we have

$$
\begin{aligned}
& r_{k}^{*}(w, \xi) \cdot \sum_{i, j=1}^{n} \widetilde{A}_{i}(w) r_{k}^{(j)}(w, \xi) S_{i j}-\sum_{i, j=1}^{n} p_{k}^{(i)}(w, \xi) r_{k}^{*}(w, \xi) \cdot r_{k}^{(j)}(w, \xi) S_{i j} \\
& \quad=\frac{1}{2} \sum_{i, j=1}^{n} p_{k}^{(i, j)}(w, \xi) S_{i j},
\end{aligned}
$$

$$
\begin{gathered}
r_{l}^{*}(w, \xi) \cdot \sum_{i, j=1}^{n} \widetilde{A_{i}}(w) r_{k}^{(j)}(w, \xi) S_{i j}-\sum_{i, j=1}^{n} p_{k}^{(i)}(w, \xi) r_{l}^{*}(w, \xi) \cdot r_{k}^{(j)}(w, \xi) S_{i j} \\
=\frac{1}{2}\left(p_{k}(w, \xi)-p_{l}(w, \xi)\right) \sum_{i, j=1}^{n} r_{l}^{*}(w, \xi) \cdot r_{k}^{(i, j)}(w, \xi) S_{i j},
\end{gathered}
$$

$k, l=1, \ldots, m, k \neq l$. Here

$$
p_{k}^{(i, j)}(w, \xi)=\frac{\partial^{2}}{\partial \xi_{i} \partial \xi_{j}} p_{k}(w, \xi), \quad \text { etc. }
$$

Now we introduce the class of functions to which our formal asymptotic solution of the initial value problem $(0.1),(0.2),(0.5)$ is presumed to belong. Let $\rho$ be real and $T>0$. We denote by $\mathscr{A}^{\rho}(T)$ the totality of the smooth functions $f(x, t, \lambda), x \in \mathbf{R}^{n}, 0 \leq t \leq T, \lambda \geq \lambda_{0}>0$, such that, when $\lambda \geq \lambda_{0}$,

$$
\sup _{x, t}\left|\partial_{t}^{k} \partial_{x}^{\alpha} f(x, t, \lambda)\right| \leq C \lambda^{k+|\alpha|-\rho}
$$

and

$$
\sup _{t}\left\|\partial_{t}^{k} f(\cdot, t, \lambda)\right\|_{s} \leq C \lambda^{k+s-\rho}
$$

for all $k=0,1,2, \ldots$, multi-indices $\alpha=\left(\alpha_{1}, \ldots, \alpha_{n}\right)$ and $s \geq 0$. Here $C$ represents various constants independent of $\lambda \geq \lambda_{0} .|\alpha|=\alpha_{1}+\cdots+\alpha_{n}$ is the length of a multi-index $\alpha$, and $\|\cdot\|_{s}$ the norm of the Sobolev space $H^{s}\left(\mathbf{R}^{n}\right)$.

Remark. We may restrict the range of $k, \alpha, s$ in bounded sets provided they allow large enough $k, s,|\alpha|$, for instance, larger than $n / 2+4$.

In order to analyze the class $\mathscr{A}^{\rho}(T)$ two auxiliary function classes $B_{\nu}(T)$, $\nu=1,2, \ldots$, and $\Phi(T)$ will be useful. $B_{\nu}(T)$ is the set of all the smooth functions $h(\sigma, x, t), \sigma=\left(\sigma_{1}, \ldots, \sigma_{\nu}\right) \in \mathbf{R}^{\nu}, x \in \mathbf{R}^{n}, 0 \leq t \leq T$, such that

$$
\sup _{\sigma, x, t}(1+|x|)^{j}\left|\partial_{x}^{\alpha} \partial_{t}^{k} \partial_{\sigma}^{\beta} h(\sigma, x, t)\right| \leq C<\infty
$$

holds for any multi-indices $\alpha=\left(\alpha_{1}, \ldots, \alpha_{n}\right), \beta=\left(\beta_{1}, \ldots, \beta_{\nu}\right), j, k=$ $0,1,2, \ldots$, with $C$ standing for various constants. The class $\Phi(T)$ consists of all the smooth functions $S(x, t), x \in \mathbf{R}^{n}, 0 \leq t \leq T$, such that, for a real $\zeta_{0}$ and a nonzero $\zeta=\left(\zeta_{1}, \ldots, \zeta_{n}\right) \in \mathbf{R}^{n}, \partial_{t} S-\zeta_{0}, \partial_{x_{j}} S-\zeta_{j}, \partial_{t}^{k} \partial_{x_{j}} S$, $j=1, \ldots, n, k=1,2, \ldots$, belong to $H^{\infty}\left(\mathbf{R}^{n}\right)=\bigcap_{s \geq 0} H^{s}\left(\mathbf{R}^{n}\right)$ uniformly with respect to $t, 0 \leq t \leq T$. 
Lemma 1.2. Let $h(\sigma, x, t) \in B_{\nu}(T)$ and $S_{1}(x, t), \ldots, S_{\nu}(x, t) \in \Phi(T)$. Then $h\left(\lambda S_{1}(x, t), \ldots, \lambda S_{\nu}(x, t), x, t\right) \in \mathscr{A}^{0}(T)$.

Proof. It is enough to consider the case $\nu=1$. The estimate (1.14) is obvious by the chain rule of differentiation of a composite function. The estimate (1.15) essentially reduces to the following

Lemma 1.3. Let $h(\sigma)$ be a smooth function of $\sigma \in \mathbf{R}$ such that all its derivatives remain bounded. Let $S(x)$ be a function $x \in \mathbf{R}^{n}$ such that for a nonzero $\zeta \in \mathbf{R}^{n}, \nabla_{x} S-\zeta \in H^{s}\left(\mathbf{R}^{n}\right)^{n}, s>n / 2$. Suppose $b(x) \in H^{s}\left(\mathbf{R}^{n}\right)$. Then $h(\lambda S(x)) b(x) \in H^{s}\left(\mathbf{R}^{n}\right)$ and

$$
\|h(\lambda S(x)) b(x)\|_{s} \leq C \lambda^{s}, \quad \lambda \geq \lambda_{0} .
$$

Proof. Let $N$ be the integer with $s \leq N<s+1$. Suppose $b(x) \in H^{N}\left(\mathbf{R}^{n}\right)$. Recall

$$
\|h(\lambda S(x)) b(x)\|_{N}^{2}=\sum_{|\alpha| \leq N}\left\|\partial_{x}^{\alpha}(h(\lambda S(x)) b(x))\right\|_{0}^{2}
$$

and

$$
\partial_{x}^{\alpha}(h(\lambda S(x)) b(x))=\sum_{\beta \leq \alpha} c_{\alpha \beta} \partial_{x}^{\beta}(h(\lambda S(x))) \partial_{x}^{\alpha-\beta} b(x),
$$

where, for $|\beta| \geq 1$,

$$
\partial_{x}^{\beta}(h(\lambda S(x)))=\sum_{k=1}^{|\beta|} \lambda^{k} h^{(k)}(\lambda S(x)) S_{\beta, k}(x)
$$

with

$$
S_{\beta, k}(x)=\sum c_{\beta^{\prime}, \ldots, \beta^{\left(k^{\prime}\right)}} \partial_{x}^{\beta^{\prime}} S(x) \cdots \partial_{x}^{\beta^{\left(k^{\prime}\right)}} S(x) .
$$

Here the summation in (1.19) is over multi-indices $\beta^{\prime}, \ldots, \beta^{\left(k^{\prime}\right)}$ such that $\beta^{\prime}+\cdots+\beta^{\left(k^{\prime}\right)} \leq \beta,\left|\beta^{\prime}\right| \geq 1, \ldots,\left|\beta^{\left(k^{\prime}\right)}\right| \geq 1$ and $\left|\beta^{\prime}\right|+\cdots+\left|\beta^{\left(k^{\prime}\right)}\right|=k$. Note $h^{(k)}(\lambda S(x))$ are uniformly bounded in $\lambda, x$. Thus, we only need to show $S_{\beta, k}(x) \partial_{x}^{\alpha-\beta} b(x) \in H^{0}\left(\mathbf{R}^{n}\right)$ for $|\beta| \geq 1$. We claim

$$
S_{\beta, k}(x) \in H^{s-k+1}\left(\mathbf{R}^{n}\right)+L^{\infty}\left(\mathbf{R}^{n}\right)
$$

for $k=1, \ldots,|\beta| \leq N$, whence

$$
\partial_{x}^{\beta}(h(\lambda S(x))) \in H^{s-|\beta|+1}\left(\mathbf{R}^{n}\right)+L^{\infty}\left(\mathbf{R}^{n}\right) .
$$

To verify (1.20), break (1.19) into three sums:

$$
S_{\beta, k}(x)=\sum^{\prime}+\sum^{\prime \prime}+\sum^{\prime \prime \prime},
$$

where $\sum^{\prime}$ is the summation over $\beta^{\prime}, \ldots, \beta^{\left(k^{\prime}\right)}$ all with length 1 , so each term consists of just $k$ factors, $\sum^{\prime \prime}$ the summation over $\beta^{\prime}, \ldots, \beta^{\left(k^{\prime}\right)}$ with $\left|\beta^{\prime}\right| \geq$ $2, \ldots,\left|\beta^{\left(k^{\prime}\right)}\right| \geq 2$, and finally $\Sigma^{\prime \prime \prime}$ one with the remaining terms. To handle $\Sigma^{\prime}$, note $\nabla S(x)=\zeta+\nabla S(x)-\zeta \in L^{\infty}\left(\mathbf{R}^{n}\right)$ by assumption. Thus, $\Sigma^{\prime} \in$ $L^{\infty}\left(\mathbf{R}^{n}\right)$. Now consider $\Sigma^{\prime \prime}$. if $\Sigma^{\prime \prime}$ contains a single factored term, then its corresponding multi-index $\beta^{\prime}$ is of length $k$ and $\partial_{x}^{\beta^{\prime}} S(x) \in H^{s-k+1}\left(\mathbf{R}^{n}\right)$. For multifactored terms, $k^{\prime} \geq 2$ and

$$
\partial_{x}^{\beta^{\prime}} S(x) \in H^{s-\left|\beta^{\prime}\right|+1}\left(\mathbf{R}^{n}\right), \ldots, \partial_{x}^{\beta^{\left(k^{\prime}\right)}} S(x) \in H^{s-\left|\beta^{\left(k^{\prime}\right)}\right|+1}\left(\mathbf{R}^{n}\right) .
$$


Since $\left(s-\left|\beta^{\prime}\right|+1\right)+\cdots+\left(s-\left|\beta^{\left(k^{\prime}\right)}\right|+1\right)-(s-k+1)=\left(k^{\prime}-1\right)(s+1) \geq s+1>n / 2$, these terms belong to $H^{s-k+1}\left(\mathbf{R}^{n}\right)$. Therefore, $\sum^{\prime \prime} \in H^{s-k+1}\left(\mathbf{R}^{n}\right)$. Finally, in handling $\Sigma^{\prime \prime \prime}$, we may discard the first derivatives of $S(x)$. If in a term there remains only one higher derivative $\partial_{x}^{\beta^{\prime}} S(x)$, then this term belongs to $H^{s-\left|\beta^{\prime}\right|+1}\left(\mathbf{R}^{n}\right) \subset H^{s-k+1}\left(\mathbf{R}^{n}\right)$. Otherwise, a similar argument to analogous cases in $\sum^{\prime \prime}$ is valid. Thus, $\Sigma^{\prime \prime \prime} \in H^{s-k+1}\left(\mathbf{R}^{n}\right)$ and (1.20) is verified. Now since $\partial_{x}^{\alpha-\beta} b(x) \in H^{N-|\alpha|+|\beta|}\left(\mathbf{R}^{n}\right)$ for $b(x) \in H^{N}\left(\mathbf{R}^{n}\right)$, and $(s-|\beta|+1)+(N-|\alpha|+$ $|\beta|)>n / 2$ for $1 \leq|\beta| \leq|\alpha| \leq N$, we have $S_{\beta, k}(x) \partial_{x}^{\alpha-\beta} b(x) \in H^{0}\left(\mathbf{R}^{n}\right)$ as we wished to show. Furthermore, in view of (1.17) and (1.18),

$$
\|h(\lambda S(x)) b(x)\|_{N} \leq C \lambda^{N}\|b(x)\|_{N}
$$

for $\lambda \geq \lambda_{0}$. On the other hand, if $b(x) \in H^{0}\left(\mathbf{R}^{n}\right)$, it is obvious that $h(\lambda S(x)) b(x)$ $\in H^{0}\left(\mathbf{R}^{n}\right)$ and

$$
\|h(\lambda S(x)) b(x)\|_{0} \leq C\|b(x)\|_{0},
$$

$\lambda \geq \lambda_{0}$. Now suppose $s<N$. Then interpolating (1.21) and (1.22) we see (1.16).

It is clear that $\mathscr{A}^{\rho}(T) \subset \mathscr{A}^{\prime}(T)$ when $\rho \geq \rho^{\prime}$. It is also clear that $\lambda^{-\tau} f(x, t, \lambda) \in \mathscr{A}^{\rho+\tau}(T)$ for $f(x, t, \lambda) \in \mathscr{A}^{\rho}(T)$. On the other hand, $B_{\nu}(T)$ is multiplicatively closed, and if $h_{1}(\sigma, x, t) \in B_{\nu}(T), h_{2}\left(\sigma^{\prime}, x, t\right) \in B_{\mu}(T)$, then the juxtaposition $h\left(\sigma, \sigma^{\prime}, x, t\right)=h_{1}(\sigma, x, t) h_{2}\left(\sigma^{\prime}, x, t\right) \in B_{\nu+\mu}(T)$. These properties will be useful in various computations of asymptotic solutions.

Now we can state our main results.

Theorem 1.4. Let $L(w)$ be the operator in the above. Assume the first characteristic field is genuinely nonlinear at $w=0$ and $\xi=\eta$, that is,

$$
\nabla_{w} p_{1}(0, \eta) \cdot r_{1}(\eta) \neq 0
$$

Suppose

$$
a_{0}(\sigma, x) \in C_{0}^{\infty}\left(\mathbf{R} \times \mathbf{R}^{n}\right)
$$

and

$$
\int_{\mathbf{R}} a_{0}(\sigma, x) d \sigma=0
$$

Then for some $T_{0}>0$ depending on $a_{0}(\sigma, x)$ and $\eta$ but independent of $\lambda \geq \lambda_{0}$ there is $U(x, t, \lambda) \in \mathscr{A}^{1}\left(T_{0}\right)$ such that

$$
\begin{gathered}
L(U) U \in \mathscr{A}^{3}\left(T_{0}\right), \\
U(x, 0, \lambda)-\lambda^{-1} a_{0}(\lambda x \cdot \eta, x) r_{1}(\eta) \in \mathscr{A}^{3}\left(T_{0}\right) .
\end{gathered}
$$

Remark. (1.27) does not contain the $t$-variable.

$U(x, t, \lambda)$ is actually the asymptotic expansion of the solution to the initial value problem $(0.1),(0.2),(0.5)$ when $n \leq 3$.

Theorem 1.5. Suppose $n=2$ or 3 . Under the same assumptions as Theorem 1.4, for some $\lambda_{1} \geq \lambda_{0}>0$ and for some $T_{1}>0$ independent of $\lambda \geq \lambda_{1}$, there is a uniquely determined $u(x, t, \lambda), \quad x \in \mathbf{R}^{n}, 0 \leq t \leq T_{1}, \lambda \geq \lambda_{0}$, such that $u(\cdot, t, \lambda) \in H^{3}\left(\mathbf{R}^{n}\right)$ and $\partial u(\cdot, t, \lambda) / \partial t \in H^{2}\left(\mathbf{R}^{n}\right)$ while $u(\cdot, t, \lambda)$ and 
$\partial u(\cdot, t, \lambda) / \partial t$ are respectively $H^{s}\left(\mathbf{R}^{n}\right)$-valued and $H^{s-1}\left(\mathbf{R}^{n}\right)$-valued continuous functions of $t \in\left[0, T_{1}\right]$ for $s<3$, and that

$$
\begin{gathered}
L(u) u=0, \\
u(x, 0, \lambda)=\lambda^{-1} a_{0}(\lambda x \cdot \eta, x) r_{1}(\eta)
\end{gathered}
$$

with

$$
\|u(\cdot, t, \lambda)-U(\cdot, t, \lambda)\|_{s} \leq C_{s} \lambda^{s-3}, \quad 0 \leq s \leq 3
$$

and

$$
\left\|\frac{\partial}{\partial t} u(\cdot, t, \lambda)-\frac{\partial}{\partial t} U(\cdot, t, \lambda)\right\|_{s} \leq C_{s} \lambda^{s-2}, \quad 0 \leq s \leq 2,
$$

uniformly with respect to $0 \leq t \leq T_{1}, \lambda \geq \lambda_{1}$. Here $C_{s}$ are positive constants independent of $\lambda \geq \lambda_{1}$.

Theorem 1.4 will be proved in $\S 2$ by an explicit construction of $U(x, t, \lambda)$. It will become clear that this is dictated essentially by the inviscid Burgers equation. However, we will have to stop before any shock develops, for smoothness of $U(x, t, \lambda)$ seems indispensable in the proof of Theorem 1.5 carried out in $\S 3$.

\section{Formal ASYMPTOTIC SOLUTION}

Let $\eta \in \mathbf{R}^{n}, \eta \neq 0$, and

$$
S_{k}(x, t)=-p_{k}(\eta) t+x \cdot \eta, \quad k=1, \ldots, m .
$$

These $S_{k}$ are phase functions corresponding to plane waves and trivially belong to the class $\Phi(T)$ for any $T>0$ (see $\S 1$ ). Our construction of the asymptotic solution $U(x, t, \lambda)$ of Theorem 1.4 follows Choquet-Bruhat [1] or Hunter and Keller [3]. However, the adoption of linear phases (2.1) and the assumptions (1.24) and particularly (1.25) will allow us to touch the third order terms of the asymptotics which are beyond the reach of general discussions due to resonance.

Now we will seek $U(x, t, \lambda)$ in the form

$$
U(x, t, \lambda)=\lambda^{-1} u_{1}(x, t, \lambda)+\lambda^{-2} u_{2}(x, t, \lambda)+\lambda^{-3} u_{3}(x, t, \lambda),
$$

where

$$
\begin{gathered}
u_{1}(x, t, \lambda)=a_{1}\left(\lambda S_{1}(x, t), x, t\right) r_{1}(\eta), \\
u_{2}(x, t, \lambda)=\sum_{k=1}^{m} b_{k k}\left(\lambda S_{k}(x, t), x, t\right) r_{k}(\eta) \\
+\sum_{k=2}^{m} b_{1 k}\left(\lambda S_{1}(x, t), x, t\right) r_{k}(\eta),
\end{gathered}
$$

and

$$
\begin{aligned}
u_{3}(x, t, \lambda)= & \sum_{\substack{k, l=1 \\
k \neq l}}^{m} c_{k l}\left(\lambda S_{k}(x, t), x, t\right) r_{l}(\eta) \\
& +\sum_{k=2}^{m} \sum_{l=1}^{m} c_{1 k l}\left(\lambda S_{1}(x, t), \lambda S_{k}(x, t), x, t\right) r_{l}(\eta)
\end{aligned}
$$


with

$$
a_{1}\left(\sigma_{1}, x, t\right), b_{j k}\left(\sigma_{j}, x, t\right), c_{k l}\left(\sigma_{k}, x, t\right) \in B_{1}\left(T_{0}\right)
$$

and

$$
c_{1 k l}\left(\sigma_{1}, \sigma_{k}, x, t\right) \in B_{2}\left(T_{0}\right)
$$

for some $T_{0}>0$.

Since we will determine $a_{1}\left(\sigma_{1}, x, t\right), \ldots, c_{1 k l}\left(\sigma_{1}, \sigma_{k}, x, t\right)$ by substituting (2.2) into (2.1), we have to study how the composite functions of $U(x, t, \lambda)$ behave. Let $A(w)$ be a decomposable $m \times m$ matrix and $A^{c}$ its constant part and $A^{d}(w)$ its rapidly decreasing part (see $\S 1$ ). We have the Taylor expansion about $w=0$ :

$$
\begin{aligned}
A(w)= & A(0)+A^{\prime}(0)[w]+\cdots+\frac{1}{k !} A^{(k)}(0)[w, \ldots, w] \\
& +R_{k+1}(A)(0, w), \quad w \in \mathbf{R}^{m}
\end{aligned}
$$

where

$$
A^{(i)}(0)[\stackrel{\urcorner}{w}, . \stackrel{i}{.}, \stackrel{\urcorner}{w}]=\left.\left(\frac{d}{d \varepsilon}\right)^{i} A(\varepsilon w)\right|_{\varepsilon=0},
$$

and $R_{k+1}(A)(0, w)$ is the remainder. Note $A(0)=A^{c}+A^{d}(0), A^{\prime}(0)[w]=$ $A^{d^{\prime}}(0)[w], \ldots, A^{(k)}(0)[w, \ldots, w]=A^{d(k)}(0)[w, \ldots, w]$, and $R_{k+1}(A)(0, w)$ $=R_{k+1}\left(A^{d}\right)(0, w)$.

Then we have the following

Lemma 2.1. Suppose $U(x, t, \lambda)$ is given by (2.2) with (2.3)-(2.7) assumed. Then the matrix

$$
R_{3}(A)(0, U)=A(U)-A(0)-A^{\prime}(0)[U]-\frac{1}{2} A^{\prime \prime}(0)[U, U]
$$

has all entries belonging to $\mathscr{A}^{3}\left(T_{0}\right)$. Furthermore,

$$
A^{\prime}(0)[U]-\lambda^{-1} A^{\prime}(0)\left[u_{1}\right]-\lambda^{-2} A^{\prime}(0)\left[u_{2}\right]
$$

and

$$
A^{\prime \prime}(0)[U, U]-\lambda^{-2} A^{\prime \prime}(0)\left[u_{1}, u_{1}\right]
$$

have all entries belonging to $\mathscr{A}^{3}\left(T_{0}\right)$.

In fact, the proof reduces to the scalar case.

Lemma 2.2. Suppose $P_{1}(x, t), \ldots, P_{\nu}(x, t) \in \Phi(T)$ and $h_{1}(\sigma, x, t), \ldots$, $h_{M}(\sigma, x, t) \in B_{\nu}(T)$ for some $T>0$. Let

$$
q(x, t, \lambda)=\sum_{k=1}^{M} \lambda^{-k} h_{k}\left(\lambda P_{1}(x, t), \ldots, \lambda P_{\nu}(x, t), x, t\right),
$$

$\lambda \geq \lambda_{0}$. If $f(r) \in \mathscr{S}(\mathbf{R})$, then

$$
f(q(x, t, \lambda))-\sum_{j=0}^{J} \frac{1}{j !} f^{(j)}(0)(q(x, t, \lambda))^{j} \in \mathscr{A}^{J+1}(T)
$$

for $J=1,2, \ldots$. 
Proof. That $(q(x, t, \lambda))^{j} \in \mathscr{A}^{j}(T), j=1,2, \ldots$, is obvious. Let

$$
R_{J+1}(f)(q)=f(q)-\sum_{j=0}^{J} \frac{1}{j !} f^{(j)}(0) q^{j}
$$

be the remainder. Since

$$
R_{J+1}(f)(0, q)=\frac{1}{J !} \int_{0}^{1}(1-\theta)^{J} f^{(J+1)}(\theta q) d \theta q^{J+1},
$$

the estimate corresponding to (1.14) is immediately obtained. To see the estimate corresponding to (1.15), note

$$
R_{J+1}(f)(0, q)=\sum_{j=J+1}^{K} \frac{1}{j !} f^{(j)}(0) q^{j}+R_{K+1}(f)(0, q)
$$

for $K>J$, and for $0 \leq s^{\prime} \leq s, s>n / 2+1$,

$$
\left\|R_{K+1}(f)(0, q)\right\|_{s^{\prime}} \leq C\left\{\left\|q^{K+1}\right\|_{s^{\prime}}+\|q\|_{(2 K+2)}^{K+1}\|q\|_{s}^{s}\right\},
$$

where $\|\cdot\|_{(p)}$ stands for the norm of $L^{p}\left(\mathbf{R}^{n}\right)$ and $C$ is positive constant depending on $K+1$ (see Yoshikawa [11]). Then since $\left\|q^{K+1}\right\|_{s^{\prime}} \leq C \lambda^{s^{\prime}-K-1},\|q\|_{s}^{s} \leq$ $C \lambda^{s^{2}}$ and $\|q\|_{(2 K+2)}^{K+1} \leq C \lambda^{-K-1}$ when $\lambda \geq \lambda_{0}$, we have

$$
\left\|R_{J+1}(f)(0, q)\right\|_{s^{\prime}} \leq C_{s^{\prime}} \lambda^{s^{\prime}-J-1}, \quad \lambda \geq \lambda_{0},
$$

by choosing $K$ large enough. The $t$-derivatives of $R_{J+1}(f)(0, q)$ can then be handled readily.

Now we derive equations governing $a_{1}\left(\sigma_{1}, x, t\right), \ldots, c_{1 k l}\left(\sigma_{1}, \sigma_{k}, x, t\right)$. Let

$$
\widetilde{L}(w)=\frac{\partial}{\partial t}+\sum_{j=1}^{n} \widetilde{A}_{j}(w) \frac{\partial}{\partial x_{j}}+\widetilde{B}(w),
$$

where $\widetilde{A_{j}}(w)=A_{0}(w)^{-1} A_{j}(w), \widetilde{B}_{j}(w)=A_{0}(w)^{-1} B(w)$. Then since $\partial U / \partial t$ and $\partial U / \partial x_{j}$ presumably belong to $\mathscr{A}^{0}\left(T_{0}\right)$, we see from Lemma 2.1 that

$$
\begin{aligned}
\frac{\partial}{\partial t} U & +\sum_{j=1}^{n}\left\{\widetilde{A}_{j}(0)+\widetilde{A}_{j}^{\prime}(0)[U]+\frac{1}{2} \widetilde{A}_{j}^{\prime \prime}(0)[U, U]\right\} \frac{\partial}{\partial x_{j}} U \\
& +\left\{\widetilde{B}(0)+\widetilde{B}^{\prime}(0)[U]\right\} U-\widetilde{L}(U) U
\end{aligned}
$$

should belong to $\mathscr{A}^{3}\left(T_{0}\right)$. Therefore, in order to realize (1.26), we have to choose $u_{1}(x, t, \lambda), u_{2}(x, t, \lambda), u_{3}(x, t, \lambda)$ in such a way that

$$
\begin{gathered}
\lambda^{-1}\left\{\frac{\partial}{\partial t} u_{1}+\sum_{j=1}^{n} \tilde{A}_{j}(0) \frac{\partial}{\partial x_{j}} u_{1}\right\} \in \mathscr{A}^{1}\left(T_{0}\right) \\
\lambda^{-1}\left\{\frac{\partial}{\partial t} u_{1}+\sum_{j=1}^{n} \tilde{A}_{j}(0) \frac{\partial}{\partial x_{j}} u_{1}+\widetilde{B}(0) u_{1}\right\} \\
+\lambda^{-2}\left\{\frac{\partial}{\partial t} u_{2}+\sum_{j=1}^{n} \widetilde{A}_{j}(0) \frac{\partial}{\partial x_{j}} u_{2}+\sum_{j=1}^{n} \widetilde{A}_{j}^{\prime}(0)\left[u_{1}\right] \frac{\partial}{\partial x_{j}} u_{1}\right\} \in \mathscr{A}^{2}\left(T_{0}\right)
\end{gathered}
$$


and

(2.15)

$$
\begin{aligned}
& \lambda^{-1}\left\{\frac{\partial}{\partial t} u_{1}+\sum_{j=1}^{n} \widetilde{A}_{j}(0) \frac{\partial}{\partial x_{j}} u_{1}+\widetilde{B}(0) u_{1}\right\} \\
& +\lambda^{-2}\left\{\frac{\partial}{\partial t} u_{2}+\sum_{j=1}^{n} \widetilde{A}_{j}(0) \frac{\partial}{\partial x_{j}} u_{2}+\widetilde{B}(0) u_{2}+\sum_{j=1}^{n} \widetilde{A_{j}^{\prime}}(0)\left[u_{1}\right] \frac{\partial}{\partial x_{j}} u_{1}\right. \\
& \left.+\widetilde{B}^{\prime}(0)\left[u_{1}\right] u_{1}\right\} \\
& +\lambda^{-3}\left\{\frac{\partial}{\partial t} u_{3}+\sum_{j=1}^{n} \widetilde{A}_{j}(0) \frac{\partial}{\partial x_{j}} u_{3}+\sum_{j=1}^{n} \widetilde{A_{j}^{\prime}}(0)\left[u_{1}\right] \frac{\partial}{\partial x_{j}} u_{2}\right.
\end{aligned}
$$

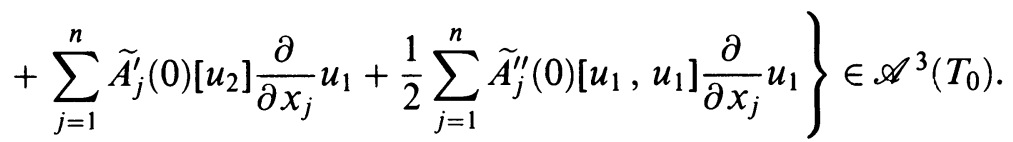

with the initial condition

$$
u_{1}(x, 0, \lambda)=a_{0}(\lambda x \cdot \eta, x) r_{1}(\eta)
$$

and

$$
u_{2}(x, 0, \lambda)=0 \text {. }
$$

By our stipulation (2.3), (2.13) is satisfied by any $a_{1}\left(\sigma_{1}, x, t\right) \in B_{1}\left(T_{0}\right)$ provided

$$
a_{1}\left(\sigma_{1}, x, 0\right)=a_{0}\left(\sigma_{1}, x\right) .
$$

Substitution of (2.3), (2.4) into (2.14) yields

$$
\begin{aligned}
& \frac{\partial}{\partial t} a_{1}\left(\sigma_{1}, x, t\right) r_{1}(\eta)+\sum_{j=1}^{n} \frac{\partial}{\partial x_{j}} a_{1}\left(\sigma_{1}, x, t\right) \widetilde{A}_{j}(0) r_{1}(\eta) \\
& \quad+a_{1}\left(\sigma_{1}, x, t\right) \widetilde{B}(0) r_{1}(\eta)+\frac{1}{2} \frac{\partial}{\partial \sigma_{1}}\left(a_{1}\left(\sigma_{1}, x, t\right)^{2}\right) \sum_{j=1}^{n} \eta_{j}{\widetilde{A_{j}^{\prime}}}_{(0)\left[r_{1}(\eta)\right] r_{1}(\eta)} \\
& \quad+\sum_{k=2}^{m} \frac{\partial}{\partial \sigma_{1}} b_{1 k}\left(\sigma_{1}, x, t\right)\left(-p_{1}(\eta)+\sum_{j=1}^{n} \eta_{j} \widetilde{A}_{j}(0)\right) r_{k}(\eta) \\
& =0
\end{aligned}
$$

where $\sigma_{1}$ is evaluated at $\sigma_{1}=S_{1}(x, t)$. Then applying Lemma 1.1, we see that $a_{1}\left(\sigma_{1}, x, t\right), b_{12}\left(\sigma_{1}, x, t\right), \ldots, b_{1 m}\left(\sigma_{1}, x, t\right)$ are governed by the equations (2.20)

$$
\begin{aligned}
\frac{\partial}{\partial t} a_{1}\left(\sigma_{1}, x, t\right)+\sum_{j=1}^{n} p_{1}^{(j)}(\eta) \frac{\partial}{\partial x_{j}} a_{1}\left(\sigma_{1}, x, t\right)+r_{1}^{*}(\eta) \cdot \widetilde{B}(0) r_{1}(\eta) a_{1}\left(\sigma_{1}, x, t\right) \\
+\frac{1}{2} \nabla_{w} p_{1}(0, \eta) \cdot r_{1}(\eta) \frac{\partial}{\partial \sigma_{1}}\left(a_{1}\left(\sigma_{1}, x, t\right)^{2}\right)=0
\end{aligned}
$$


and

$$
\begin{aligned}
\frac{\partial}{\partial \sigma_{1}} b_{1 k}\left(\sigma_{1}, x, t\right)= & \sum_{j=1}^{n} r_{k}^{*}(\eta) \cdot r_{1}^{(j)}(\eta) \frac{\partial}{\partial x_{j}} a_{1}\left(\sigma_{1}, x, t\right) \\
& +\frac{1}{2} r_{k}^{*}(\eta) \cdot\left(\nabla_{w} r_{1}(0, \eta) \cdot r_{1}(\eta)\right) \frac{\partial}{\partial \sigma_{1}}\left(a_{1}\left(\sigma_{1}, x, t\right)^{2}\right) \\
& +\frac{1}{p_{1}(\eta)-p_{k}(\eta)} r_{k}^{*}(\eta) \cdot \widetilde{B}(0) r_{1}(\eta) a_{1}\left(\sigma_{1}, x, t\right)
\end{aligned}
$$

$k=2, \ldots, m$. Note

$$
\left\{\frac{\partial}{\partial t}+\sum_{j=1}^{n} p_{1}^{(j)}(\eta) \frac{\partial}{\partial x_{j}}\right\} S_{1}(x, t)=0 .
$$

Lemma 2.3. Suppose (1.23) holds. Let $a_{0}\left(\sigma_{1}, x\right)$ satisfy (1.24) and (1.25). Then for some $T_{0}>0$ there is a unique $a_{1}\left(\sigma_{1}, x, t\right) \in B_{1}\left(T_{0}\right)$ satisfying the equation (2.20) under the initial condition (2.18). Furthermore, $a_{1}\left(\sigma_{1}, x, t\right)$ is compactly supported with respect to $\sigma_{1}, x$ and

$$
\int_{\mathbf{R}} a_{1}\left(\sigma_{1}, x, t\right) d \sigma_{1}=0
$$

is valid.

In fact, let $X(t)=\left(x_{1}+p_{1}^{(1)}(\eta) t, \ldots, x_{n}+p_{1}^{(n)}(\eta) t\right)$ and

$$
\tilde{a}_{1}\left(\sigma_{1}, t\right)=a_{1}\left(\sigma_{1}, X(t), t\right) .
$$

Then (2.20) essentially reduces to the inviscid Burgers equation (see Lax [8]):

$$
\frac{\partial}{\partial t} \tilde{a}_{1}\left(\sigma_{1}, t\right)+G \frac{\partial}{\partial \sigma_{1}}\left(\frac{1}{2} \tilde{a}\left(\sigma_{1}, t\right)^{2}\right)+H \tilde{a}\left(\sigma_{1}, t\right)=0
$$

with $G=\nabla_{w} p_{1}(0, \eta) \cdot r_{1}(\eta) \neq 0$ by $(1.23)$ and $H=r_{1}^{*}(\eta) \cdot \widetilde{B}(0) r_{1}(\eta)$. Since $a_{0}\left(\sigma_{1}, x\right)$ is smooth and compactly supported, we can choose $T_{0}>0$ such that $a_{1}\left(\sigma_{1}, x, t\right)$, uniquely solved from (2.20), (2.18) for $0 \leq t \leq T_{0}$, does not develop any shock. In other words, the method of characteristics works for $0 \leq t \leq T_{0}$, and $a_{1}\left(\sigma_{1}, x, t\right)$ is easily seen to be compactly supported in $\sigma_{1}$ and $x$. To verify (2.23), consider the linear differential equation satisfied by $\int_{\mathbf{R}} a_{1}(\sigma, x, t) d \sigma_{1}$ with the vanishing initial data because of (1.25).

Corollary 2.4. Under the assumption of Lemma 2.3, there are $b_{1 k}\left(\sigma_{1}, x, t\right) \in$ $B_{1}\left(T_{0}\right), k=2, \ldots, m$, with compact support in $\sigma_{1}$ and $x$.

In fact, consider (2.21). For each $k$, the right-hand side is compactly supported in $\sigma_{1}$ and $x$. Furthermore, its integral with respect to $\sigma_{1}$ over the whole line vanishes because of $(2.23)$. Hence, we have $b_{1 k}\left(\sigma_{1}, x, t\right)$ compactly supported in $\sigma_{1}$ and $x$.

Remark. Such $b_{1 k}\left(\sigma_{1}, x, t\right)$ are uniquely determined.

Recall the initial condition (2.17). This leads to the following initial condition on $b_{k k}\left(\sigma_{k}, x, t\right), k=1, \ldots, m$. Namely, we require

$$
b_{11}\left(\sigma_{1}, x, 0\right)=0 \text {, }
$$




$$
b_{k k}\left(\sigma_{k}, x, 0\right)=-b_{1 k}\left(\sigma_{k}, x, 0\right), \quad k=2, \ldots, m .
$$

To determine $b_{k k}\left(\sigma_{k}, x, t\right), \quad k=1, \ldots, m$, we appeal to (2.15). Let $\tilde{u}_{2}\left(\sigma_{1}, x, t\right)=\sum_{k=2}^{m} b_{1 k}\left(\sigma_{1}, x, t\right) r_{k}(\eta)$, a known vector, compactly supported in $\sigma_{1}$ and $x$. Then (2.15) yields

(2.26)

$$
\begin{aligned}
& \sum_{k=1}^{m}\left\{\frac{\partial}{\partial t} b_{k k}\left(\sigma_{k}, x, t\right) r_{k}(\eta)+\sum_{j=1}^{n} \frac{\partial}{\partial x_{j}} b_{k k}\left(\sigma_{k}, x, t\right) \tilde{A}_{j}(0) r_{k}(\eta)\right. \\
& +b_{k k}\left(\sigma_{k}, x, t\right) \widetilde{B}(0) r_{k}(\eta) \\
& +a_{1}\left(\sigma_{1}, x, t\right) \frac{\partial}{\partial \sigma_{k}} b_{k k}\left(\sigma_{k}, x, t\right) \sum_{j=1}^{n} \eta_{j} \widetilde{A}_{j}^{\prime}(0)\left[r_{1}(\eta)\right] r_{k}(\eta) \\
& \left.+\frac{\partial}{\partial \sigma_{1}} a_{1}\left(\sigma_{1}, x, t\right) b_{k k}\left(\sigma_{k}, x, t\right) \sum_{j=1}^{n} \eta_{j}{\widetilde{A_{j}^{\prime}}}_{j}(0)\left[r_{k}(\eta)\right] r_{1}(\eta)\right\} \\
& +\frac{1}{2} a_{1}\left(\sigma_{1}, x, t\right)^{2} \frac{\partial}{\partial \sigma_{1}} a_{1}\left(\sigma_{1}, x, t\right) \sum_{j=1}^{n} \eta_{j} \widetilde{A_{j}^{\prime \prime}}(0)\left[r_{1}(\eta), r_{1}(\eta)\right] r_{1}(\eta) \\
& +\sum_{j=1}^{n} a_{1}\left(\sigma_{1}, x, t\right) \frac{\partial}{\partial x_{j}} a_{1}\left(\sigma_{1}, x, t\right) \widetilde{A_{j}^{\prime}}(0)\left[r_{1}(\eta)\right] r_{1}(\eta) \\
& +\frac{\partial}{\partial t} \tilde{u}_{2}\left(\sigma_{1}, x, t\right)+\sum_{j=1}^{n} \tilde{A}_{j}(0) \frac{\partial}{\partial x_{j}} \tilde{u}_{2}\left(\sigma_{1}, x, t\right)+\widetilde{B}(0) \tilde{u}_{2}\left(\sigma_{1}, x, t\right) \\
& +a_{1}\left(\sigma_{1}, x, t\right) \sum_{j=1}^{n} \eta_{j} \widetilde{A}_{j}^{\prime}(0)\left[r_{1}(\eta)\right] \frac{\partial}{\partial \sigma_{1}} \tilde{u}_{2}\left(\sigma_{1}, x, t\right) \\
& +\frac{\partial}{\partial \sigma_{1}} a_{1}\left(\sigma_{1}, x, t\right) \sum_{j=1}^{n} \eta_{j}{\widetilde{A_{j}^{\prime}}}_{j}(0)\left[\tilde{u}_{2}\left(\sigma_{1}, x, t\right)\right] r_{1}(\eta) \\
& +\sum_{\substack{k, l=1 \\
k \neq l}}^{m}\left(-p_{k}(\eta)+p_{l}(\eta)\right) \frac{\partial}{\partial \sigma_{k}} c_{k l}\left(\sigma_{k}, x, t\right) r_{l}(\eta) \\
& +\sum_{k=2}^{m} \sum_{l=1}^{m}\left\{\left(-p_{1}(\eta)+p_{l}(\eta)\right) \frac{\partial}{\partial \sigma_{1}}+\left(-p_{k}(\eta)+p_{l}(\eta)\right) \frac{\partial}{\partial \sigma_{k}}\right\} \\
& \cdot c_{1 k l}\left(\sigma_{1}, \sigma_{k}, x, t\right) r_{l}(\eta) \\
& =0 \text {. }
\end{aligned}
$$

Now, for each $k=1, \ldots, m$, consider the coefficient of $r_{k}(\eta)$. We then let the sum of the terms containing only $\sigma_{k}$ vanish. We thus stipulate (2.27)

$$
\begin{gathered}
\frac{\partial}{\partial t} b_{11}\left(\sigma_{1}, x, t\right)+\sum_{j=1}^{n} p_{1}^{(j)}(\eta) \frac{\partial}{\partial x_{j}} b_{11}\left(\sigma_{1}, x, t\right)+r_{1}^{*}(\eta) \cdot \widetilde{B}(0) r_{1}(\eta) b_{11}\left(\sigma_{1}, x, t\right) \\
+\nabla_{w} p_{1}(0, \eta) \cdot r_{1}(\eta) \frac{\partial}{\partial \sigma_{1}}\left(a_{1}\left(\sigma_{1}, x, t\right) b_{11}\left(\sigma_{1}, x, t\right)\right)=R\left(\sigma_{1}, x, t\right)
\end{gathered}
$$


where

$$
\begin{array}{r}
R\left(\sigma_{1}, x, t\right)=-\left\{\frac{1}{6} r_{1}^{*}(\eta) \cdot \sum_{j=1}^{n} \eta_{j}{\widetilde{A_{j}^{\prime \prime}}}_{j}(0)\left[r_{1}(\eta), r_{1}(\eta)\right] r_{1}(\eta) \frac{\partial}{\partial \sigma_{1}}\left(a_{1}\left(\sigma_{1}, x, t\right)^{3}\right)\right. \\
+\frac{1}{2} \sum_{j=1}^{n} r_{1}^{*}(\eta) \cdot \widetilde{A}_{j}^{\prime}(0)\left[r_{1}(\eta)\right] r_{1}(\eta) \frac{\partial}{\partial x_{j}}\left(a_{1}\left(\sigma_{1}, x, t\right)^{2}\right)+r_{1}^{*}(\eta) \\
\quad\left(\frac{\partial}{\partial t} \tilde{u}_{2}\left(\sigma_{1}, x, t\right)+\sum_{j=1}^{n} \widetilde{A}_{j}(0) \frac{\partial}{\partial x_{j}} \tilde{u}_{2}\left(\sigma_{1}, x, t\right)\right. \\
+\widetilde{B}(0) \tilde{u}_{2}\left(\sigma_{1}, x, t\right) \\
+a_{1}\left(\sigma_{1}, x, t\right) \sum_{j=1}^{n} \eta_{j}{\widetilde{A_{j}^{\prime}}}_{j}(0)\left[r_{1}(\eta)\right] \frac{\partial}{\partial \sigma_{1}} \tilde{u}_{2}\left(\sigma_{1}, x, t\right) \\
\left.\left.\quad+\frac{\partial}{\partial \sigma_{1}} a_{1}\left(\sigma_{1}, x, t\right) \sum_{j=1}^{n} \eta_{j} \tilde{A}_{j}^{\prime}(0)\left[\tilde{u}_{2}\left(\sigma_{1}, x, t\right)\right] r_{1}(\eta)\right)\right\} .
\end{array}
$$

For $k=2, \ldots, m$, we stipulate

$$
\begin{aligned}
\frac{\partial}{\partial t} b_{k k}\left(\sigma_{k}, x, t\right) & +\sum_{j=1}^{n} p_{k}^{(j)}(\eta) \frac{\partial}{\partial x_{j}} b_{k k}\left(\sigma_{k}, x, t\right) \\
& +r_{k}^{*}(\eta) \cdot \widetilde{B}(0) r_{k}(\eta) b_{k k}\left(\sigma_{k}, x, t\right)=0 .
\end{aligned}
$$

Here we have employed Lemma 1.1 as we did before in deriving (2.20) and (2.21).

Lemma 2.5. We have $b_{k k}\left(\sigma_{k}, x, t\right) \in B_{1}\left(T_{0}\right), k=1, \ldots, m$. Each $b_{k k}\left(\sigma_{k}, x, t\right)$ is compactly supported in $\sigma_{k}$ and $x$.

In fact, for $k \geq 2$, this is obvious from (2.29) and (2.25) since $b_{1 k}\left(\sigma_{k}, x, 0\right)$ is compactly supported in $\sigma_{k}$ and $x$. On the other hand, for $b_{11}\left(\sigma_{1}, x, t\right)$, since $R\left(\sigma_{1}, x, t\right)$ vanishes for large $\left|\sigma_{1}\right|,(2.27)$ and (2.24) imply $b_{11}\left(\sigma_{1}, x, t\right)$ $\in B_{1}\left(T_{0}\right)$ with compact support in $\sigma_{1}$ and $x$.

Let us return to (2.26). The terms involving $a_{1}\left(\sigma_{1}, x, t\right), b_{11}\left(\sigma_{1}, x, t\right), \ldots$, $b_{m m}\left(\sigma_{m}, x, t\right)$, and $\tilde{u}_{2}\left(\sigma_{1}, x, t\right)$, which still remain in (2.26), have the form

$$
\sum_{k=1}^{m} \sum_{\substack{l=1 \\ k \neq l}}^{m} C_{k l}\left(\sigma_{k}, x, t\right) r_{l}(\eta)+\sum_{k=2}^{m} \sum_{l=1}^{m} C_{1 k l}\left(\sigma_{1}, \sigma_{k}, x, t\right) r_{l}(\eta)
$$

with $C_{k l}\left(\sigma_{k}, x, t\right) \in B_{1}\left(T_{0}\right), C_{1 k l}\left(\sigma_{1}, \sigma_{k}, x, t\right) \in B_{2}\left(T_{0}\right)$. Furthermore, $C_{k l}\left(\sigma_{k}, x, t\right)$ are compactly supported in $\sigma_{k}, x$, and $C_{1 k l}\left(\sigma_{1}, \sigma_{k}, x, t\right)$ are so in $\sigma_{1}, \sigma_{k}, x$. Therefore, we can solve

$$
c_{k l}\left(\sigma_{k}, x, t\right) \in B_{1}\left(T_{0}\right) \text { and } c_{1 k l}\left(\sigma_{1}, \sigma_{k}, x, t\right) \in B_{2}\left(T_{0}\right)
$$

from the equations

$$
\left(-p_{k}(\eta)+p_{l}(\eta)\right) \frac{\partial}{\partial \sigma_{k}} c_{k l}\left(\sigma_{k}, x, t\right)+C_{k l}\left(\sigma_{k}, x, t\right)=0
$$


$k, l=1, \ldots, m, k \neq l$, and

$$
\begin{gathered}
\left\{\left(-p_{1}(\eta)+p_{l}(\eta)\right) \frac{\partial}{\partial \sigma_{1}}+\left(-p_{k}(\eta)+p_{l}(\eta)\right) \frac{\partial}{\partial \sigma_{k}}\right\} c_{1 k l}\left(\sigma_{1}, \sigma_{k}, x, t\right) \\
+C_{1 k l}\left(\sigma_{1}, \sigma_{k}, x, t\right)=0, \\
k=2, \ldots, m, l=1, \ldots, m .
\end{gathered}
$$

Remark. $u_{3}(x, t, \lambda)$ is computed only to realize

$$
\widetilde{L}(U) U \in\left(\mathscr{A}_{3}\left(T_{0}\right)\right)^{m} .
$$

$u_{3}(x, t, \lambda)$ does not necessarily include all the terms of possible third order terms in the expansion of $U=U(x, t, \lambda)$. Also compare with (1.30) and (1.31).

Finally note

$$
L(U) U=A_{0}(U) \widetilde{L}(U) U=\left(A_{0}(0)+A_{0}^{\prime}(0)[U]+\cdots\right) \widetilde{L}(U) U \in \mathscr{A}^{3}\left(T_{0}\right) .
$$

Thus, we have proved Theorem 1.4.

\section{REMAINDER ESTIMATES}

Now we proceed to a proof of Theorem 1.5. Let $U(x, t, \lambda) \in \mathscr{A}^{1}\left(T_{0}\right)$ be the asymptotic solution constructed in the previous section. Let

$$
F(x, t, \lambda)=-L(U(x, t, \lambda)) U(x, t, \lambda)
$$

and

$$
v_{0}(x, \lambda)=\lambda^{-1} a_{0}(\lambda \eta \cdot x, x) r_{1}(\eta)-U(x, 0, \lambda) .
$$

So far, we have shown $F(x, t, \lambda) \in \mathscr{A}^{3}\left(T_{0}\right)$ and $v_{0}(x, \lambda) \in \mathscr{A}^{3}\left(T_{0}\right)$. Thus, in particular, for $0 \leq s \leq 3$,

$$
\sup _{0 \leq t \leq T_{0}}\|F(\cdot, t, \lambda)\|_{s} \leq L \lambda^{s-3}
$$

and

$$
\left\|v_{0}(\cdot, \lambda)\right\|_{s} \leq M \lambda^{s-3}
$$

for $\lambda \geq \lambda_{0}$. Here $L$ and $M$ are positive constants independent of $\lambda \geq \lambda_{0}$.

Now we wish to find an $m$-vector-valued function $v(x, t, \lambda)$, which satisfies the equation

$$
L(U+v)(U+v)=0,
$$

valid in a time interval $0 \leq t \leq T_{1} \leq T_{0}, \lambda \geq \lambda_{1}$, and

$$
v(x, 0, \lambda)=v_{0}(x, \lambda), \quad \lambda \geq \lambda_{1} .
$$

Here $\lambda_{1}$ is large enough and $T_{1}$ is independent of $\lambda \geq \lambda_{1}$.

Let us rewrite the equation (3.5) in a more convenient form. Suppose $w \in$ $\mathbf{R}^{m}$. Let

$$
\begin{aligned}
C(U, w) v= & \sum_{j=0}^{n} \int_{0}^{1} A_{j}^{\prime}(U+\theta w)[v] d \theta \frac{\partial U}{\partial x_{j}} \\
& +\int_{0}^{1} B^{\prime}(U+\theta w)[v] d \theta U .
\end{aligned}
$$


Here $x_{0}$ stands for $t, x_{0}=t$. Denote by $L_{U}(w)$ the operator

$$
L_{U}(w) v=L(U+w) v+C(U, w) v,
$$

acting on $m$-vector-valued functions $v=v(x, t, \lambda)$. Then, in view of (3.1), (3.5) turns out to be

$$
L_{U}(v) v=F .
$$

We solve the initial value problem (3.9), (3.6) by a routine iteration method. Namely, let $w^{0}(x, t, \lambda)=0$ and generally solve $w^{k}(x, t, \lambda), k=1,2,3, \ldots$, from

$$
L_{U}\left(w^{k-1}\right) w^{k}=F, \quad w^{k}(x, 0, \lambda)=v_{0}(x, \lambda) .
$$

What we have to show is that these $w^{k}(x, 0, \lambda)$ satisfy appropriate a priori estimates and that with a suitable choice of $\lambda_{1}$ and $T_{1}>0$ these $w^{k}(x, t, \lambda)$ converge in certain Sobolev spaces over $\mathbf{R}^{2}$ or $\mathbf{R}^{3}$ uniformly with respect to $0 \leq t \leq T_{1}$ while with a definite control over the order in $\lambda$.

On the other hand, because of (3.7), (3.8), (3.10) and smoothness of $U(x, t, \lambda)$, note that $w^{k}(x, t, \lambda)$, which are solutions of linear hyperbolic problems, are smooth in their arguments, up to $t \leq T_{0}$.

Lemma 3.1. Let $n=2$ or 3. Suppose $w(x, t, \lambda)$ is a smooth $m$-vector-valued function defined for $x \in \mathbf{R}^{n}, 0 \leq t \leq T_{0}, \lambda \geq \lambda_{0}$, such that

$$
\lambda^{3-s}\|w(\cdot, t, \lambda)\|_{s} \leq N, \quad s=0,1,2,3,
$$

and

$$
\lambda^{2-s}\left\|\frac{\partial}{\partial t} w(\cdot, t, \lambda)\right\|_{s} \leq N_{1}, \quad s=0,1,2,
$$

for $0 \leq t \leq T_{0}, \lambda \geq \lambda_{0}, N, N_{1}$ being constants independent of $t, \lambda$. Let $v(x, t, \lambda)$ be the solution of the initial value problem:

$$
\begin{gathered}
L_{U}(w(x, t, \lambda)) v(x, t, \lambda)=F(x, t, \lambda), \\
v(x, 0, \lambda)=v_{0}(x, \lambda) .
\end{gathered}
$$

Then there is a positive function $K_{0}(p, q, \lambda)$ of $p \geq 0, q \geq 0, \lambda \geq \lambda_{0}$, and $a$ positive constant $C_{0}$ such that

$$
\|v(\cdot, t, \lambda)\|_{0} \leq C_{0} e^{K_{0}\left(N, N_{1}, \lambda\right) t}\left(M^{2}+L^{2} t\right)^{1 / 2} \lambda^{-3}
$$

for $0 \leq t \leq T_{0}, \lambda \geq \lambda_{0}$.

Proof. We compute the $L^{2}$-inner product $\left(v, L_{U}(w) v\right)$. Then

$$
\begin{aligned}
\frac{d}{d t}\left(v, A_{0}(U+w) v\right)= & 2(v, F)+\left(v, \sum_{j=0}^{n} \frac{\partial}{\partial x_{j}}\left(A_{j}(U+w)\right) v\right) \\
& -2(v,(B(U+w)+C(U, w)) v) .
\end{aligned}
$$

Before estimating the right-hand side, observe that for a decomposable matrix $A(\cdot)$ the Taylor expansion yields

$$
\begin{aligned}
A(U+w)= & A(w)+A^{\prime}(w)[U]+\cdots+\frac{1}{k !} A^{(k)}(w)[U, \ldots, U] \\
& +R_{k+1}(A)(w, U)
\end{aligned}
$$


for any $k$. Here $A^{(i)}(w)[U, \ldots, U]=\left.(d / d \varepsilon)^{i} A(w+\varepsilon U)\right|_{\varepsilon=0}$ (cf. (2.9)) and $R_{k+1}(A)$ is the remainder. In our present situation, we have

$$
\left\|R_{k+1}(A)(w, U)\right\|_{s} \leq C_{k}\left(1+\|w(\cdot, t, \lambda)\|_{3}^{4}\right) \lambda^{5-k}
$$

for $0 \leq s \leq 3, \lambda \geq \lambda_{0}$ and $\lambda \geq \lambda_{0}$. For $i=1, \ldots, k$, the entries of $A^{(i)}(w)[U, \ldots, U]$ are sums of terms of the form $U_{l}^{(i)} w_{l}^{(i)}$ or $U_{l}^{(i)}$ with $U_{l}^{(i)}$, $U_{l}^{\prime(i)} \in \mathscr{A}^{i}\left(T_{0}\right)$ and

$$
\begin{gathered}
\left\|w_{l}^{(i)}(\cdot, t, \lambda)\right\|_{s} \leq C_{l}^{(i)}\left(\|w(\cdot, t, \lambda)\|_{3}\right)\|w(\cdot, t, \lambda)\|_{s}, \\
\left\|\frac{\partial}{\partial t} w_{l}^{(i)}(\cdot, t, \lambda)\right\|_{s} \leq C_{l 1}^{(i)}\left(\|w(\cdot, t, \lambda)\|_{3}\right)\left\|\frac{\partial}{\partial t} w(\cdot, t, \lambda)\right\|_{s}
\end{gathered}
$$

for $\lambda \geq \lambda_{0}, 0 \leq t \leq T_{0}$. Here $C_{l}^{(i)}(p)$ and $C_{l 1}^{(i)}(p)$ are positive nondecreasing functions of $p \geq 0$. As for the first term $A(w)$ in (3.16), the entries of the rapidly decreasing part $A^{d}(w)-A^{d}(0)$ satisfy similar estimates to (3.18), (3.19) (cf. [11]). $A_{0}(U+w), \ldots, A_{n}(U+w), B(U+w)$ fall in this observation, and $C(U, w)$ can be handled using (3.7). Then it is not difficult to see the estimate

$$
\begin{aligned}
\mid(v, & \left.\sum_{j=0}^{n} \frac{\partial}{\partial x_{j}}\left(A_{j}(U+w)\right) v\right)-2(v,(B(U+w)+C(U, w)) v) \mid \\
\leq & \left\{C_{1}+C_{2}\left(\|w(\cdot, t, \lambda)\|_{3}\right)\left(\|w(\cdot, t, \lambda)\|_{s}+\left\|\frac{\partial}{\partial t} w(\cdot, t, \lambda)\right\|_{s-1}\right)\right\} \\
& \cdot\left(v, A_{0}(U+w) v\right)
\end{aligned}
$$

for $1+n / 2<s<3$. Here $C_{1}$ is a constant, $C_{2}(p)$ a nondecreasing positive function of $p \geq 0$. On the other hand, from (1.2), (3.3),

$$
\begin{aligned}
2|(v, F)| & \leq 2\left(v, A_{0}(U+w) v\right)^{1 / 2}\left(F, A_{0}(U+w)^{-1} F\right)^{1 / 2} \\
& \leq\left(v, A_{0}(U+w) v\right)+\frac{1}{\gamma} L^{2} \lambda^{-6} .
\end{aligned}
$$

Thus, because of (3.11) and (3.12), we have

$$
\frac{d}{d t}\left(v, A_{0}(U+w) v\right) \leq 2 K_{0}\left(N, N_{1}, \lambda\right)\left(v, A_{0}(U+w) v\right)+\frac{1}{\gamma} L^{2} \lambda^{-6},
$$

where

$$
2 K_{0}\left(N, N_{1}, \lambda\right)=1+C_{1}+C_{2}(N)\left(N+N_{1}\right) \lambda^{s-3} .
$$

Gronwall's inequality together with (1.2) imply (3.15) with

$$
C_{0}=\left(\max \left(\Gamma / \gamma, 1 / \gamma^{2}\right)\right)^{1 / 2} \text {. }
$$

Next we estimate $\langle D\rangle^{2} v(x, t, \lambda),\langle D\rangle^{2}=I-\Delta_{x}$, where $\Delta_{x}=\partial_{1}^{2}+\cdots+\partial_{n}^{2}$ is the Laplacian on $\mathbf{R}^{n}$. First we derive the equation satisfied by $\langle D\rangle^{2} v$. Let

$$
L_{U, 2}(w)=L_{U}(w)-2 \sum_{j=1}^{n} A_{0}(U+w) \nabla_{x}\left(\tilde{A}_{j}(U+w)\right) \cdot \nabla_{x} \frac{\partial}{\partial x_{j}}\langle D\rangle^{-2}
$$


where $\tilde{A_{j}}=A_{0}^{-1} A_{j}, \nabla_{x}$ is the gradient operation, and $\langle D\rangle^{-2}$ is a pseudodifferential operator. Let

$$
\begin{aligned}
F_{2}(x, t, \lambda)= & A_{0}(U+w)\langle D\rangle^{2}\left(A_{0}(U+w)^{-1} F(x, t, \lambda)\right) \\
& +\sum_{j=1}^{n} \Phi_{j}(U, w) \frac{\partial v}{\partial x_{j}}+\Psi(U, w) v
\end{aligned}
$$

where

$$
\begin{aligned}
\Phi_{j}(U, w)= & A_{0}(U+w) \Delta_{x}\left(\tilde{A}_{j}(U+w)\right) \\
& +2 A_{0}(U+w) \frac{\partial}{\partial x_{j}}\left(A_{0}(U+w)^{-1}(B(U+w)+C(U, w))\right)
\end{aligned}
$$

and

$$
\Psi(U, w)=A_{0}(U+w) \Delta_{x}\left(A_{0}(U+w)^{-1}(B(U+w)+C(U, w))\right) .
$$

Then by a straightforward computation, we see

$$
L_{U, 2}(w)\langle D\rangle^{2} v=F_{2}
$$

Lemma 3.2. Under the same assumptions as Lemma 3.1, there are positive functions $K_{2}(p, q, \lambda)$ and $K_{20}(p, q), K_{21}(p, q)$, of $p \geq 0, q \geq 0, \lambda \geq \lambda_{0}$, such that

$$
\begin{aligned}
& \|v(\cdot, t, \lambda)\|_{2} \\
& \quad \leq C_{0} e^{K_{2}\left(N, N_{1}, \lambda\right) t}\left(M^{2}+\left(K_{20}\left(N, N_{1}\right) M^{2}+K_{21}\left(N, N_{1}\right) L^{2}\right) t\right)^{1 / 2} \lambda^{-1}
\end{aligned}
$$

for $\lambda \geq \lambda_{0}, 0 \leq t \leq T_{0}$.

Proof. Computing the inner product $\left(\langle D\rangle^{2} v, L_{U, 2}(w)\langle D\rangle^{2} v\right)$, we obtain (3.25)

$$
\begin{aligned}
& \frac{d}{d t}\left(\langle D\rangle^{2} v, A_{0}(U+w)\langle D\rangle^{2} v\right) \\
& \leq\left\{C_{1}^{\prime}+C_{2}^{\prime}\left(\|w\|_{3}\right)\left(\|w\|_{s}+\left\|\frac{\partial}{\partial t} w\right\|_{s-1}\right)\right\}\left(\langle D\rangle^{2} v, A_{0}(U+w)\langle D\rangle^{2} v\right) \\
& \quad+2\left|\left(\langle D\rangle^{2} v, F_{2}\right)\right|
\end{aligned}
$$

where $C_{1}^{\prime}$ is a positive constant and $C_{2}^{\prime}(p)$ a positive nondecreasing function of $p \geq 0$. To evaluate $\left(\langle D\rangle^{2} v, F_{2}\right)$, we see from (3.22), (3.16), (3.17), (3.18) that $\bar{\Phi}_{j}(U, w)$ and $\Psi(U, w)$ can be written as

$$
\Phi_{j}(U, w)=\sum_{k=0}^{3} \Phi_{j}^{(k)}(U, w)
$$

and

$$
\Psi(U, w)=\sum_{k=0}^{3} \Psi^{(k)}(U, w)
$$

where, for $0 \leq s \leq 1$,

$$
\left\|\Phi_{j}^{(0)}(U, w)\right\|_{s} \leq C_{j}^{(0)}\left(\|w(\cdot, t, \lambda)\|_{3}\right)\|w(\cdot, t, \lambda)\|_{2+s}
$$


while $\Phi_{j}^{(k)}(U, w), k \geq 1$, are finite sums of terms of the form $U_{j l}^{(k)} w_{j l}^{(k)}$ with

$$
\left\|w_{j l}^{(k)}\right\|_{s} \leq C_{j}^{(k)}\left(\|w(\cdot, t, \lambda)\|_{3}\right)\|w(\cdot, t, \lambda)\|_{s-k+3},
$$

$0 \leq s \leq k, k=1,2,3$, for $\lambda \geq \lambda_{0}, 0 \leq t \leq T_{0}$, and $U_{j l}^{(k)} \in \mathscr{A}^{2-k}\left(T_{0}\right)$. Here $C_{j}^{(k)}(p)$ are nondecreasing functions of $p \geq 0, k=0,1,2,3$. Similarly,

$$
\left\|\Psi^{(0)}(U, w)\right\|_{s} \leq C^{(0)}\left(\|w(\cdot, t, \lambda)\|_{3}\right)\|w(\cdot, t, \lambda)\|_{2+s},
$$

$0 \leq s \leq 1$, and $\Psi^{(k)}(U, w), k=1,2,3$, are finite sums of terms of the form $U_{l}^{(k)} w_{l}^{(k)}$ with

$$
\left\|w_{l}^{(k)}\right\|_{s} \leq C^{(k)}\left(\|w(\cdot, t, \lambda)\|_{3}\right)\|w(\cdot, t, \lambda)\|_{s-k+3},
$$

$0 \leq s \leq k, k=1,2,3$, and $U_{l}^{(k)} \in \mathscr{A}^{1-k}\left(T_{0}\right)$. Now we have, for $n / 2-1<$ $s<1$,

$$
\left|\sum_{j=1}^{n}\left(\langle D\rangle^{2} v, \Phi_{j}(U, w) \frac{\partial v}{\partial x_{j}}\right)\right| \leq C_{2}^{\prime \prime}\left(\|w\|_{3}\right)\|w\|_{2+s}\left(\|v\|_{2}^{2}+\lambda\|v\|_{1}\|v\|_{2}\right)
$$

for $\lambda \geq \lambda_{0}, 0 \leq t \leq T_{0}$ with a nondecreasing function $C_{2}^{\prime \prime}(p)$ of $p \geq 0$. In fact, note

$$
\left(\langle D\rangle^{2} v, \Phi_{j}(U, w) \frac{\partial v}{\partial x_{j}}\right)=\sum_{k=0}^{3}\left(\langle D\rangle^{2} v, \Phi_{j}^{(k)}(U, w) \frac{\partial v}{\partial x_{j}}\right)
$$

and, for $n / 2-1<s<1$,

$$
\begin{aligned}
\left|\left(\langle D\rangle^{2} v, \Phi_{j}^{(0)}(U, w) \frac{\partial v}{\partial x_{j}}\right)\right| & \leq C\|v\|_{2}\left\|\Phi_{j}^{(0)}\right\|_{s}\left\|\frac{\partial v}{\partial x_{j}}\right\|_{1} \\
& \leq C C_{j}^{(0)}\left(\|w\|_{3}\right)\|w\|_{2+s}\|v\|_{2}^{2}, \\
\left|\left(\langle D\rangle^{2} v, \Phi_{j}^{(1)}(U, w) \frac{\partial v}{\partial x_{j}}\right)\right| & \leq \sum\|v\|_{2}\left\|U_{j l}^{(1)}\right\|_{(\infty)}\left\|w_{j l}^{(1)} \frac{\partial v}{\partial x_{j}}\right\|_{0} \\
& \leq C \lambda^{-1} C_{j}^{(1)}\left(\|w\|_{3}\right)\|w\|_{2+s}\|v\|_{2}^{2}, \\
\left|\left(\langle D\rangle^{2} v,\left(\Phi_{j}^{(2)}(U, w)+\Phi_{j}^{(3)}(U, w)\right) \frac{\partial v}{\partial x_{j}}\right)\right| & \leq C\left(C_{j}^{(2)}\left(\|w\|_{3}\right)+\lambda C_{j}^{(3)}\left(\|w\|_{3}\right)\right)\|w\|_{2+s}\|v\|_{2}\|v\|_{1} .
\end{aligned}
$$

Similarly, we have, for $n / 2-1<s<1$,

$$
\left|\left(\langle D\rangle^{2} v, \Psi(U, w) v\right)\right| \leq C_{2}^{\prime \prime \prime}\left(\|w\|_{3}\right)\|w\|_{2+s}\left(\|v\|_{2}\|v\|_{1}+\lambda^{2}\|v\|_{2}\|v\|_{0}\right)
$$

for $\lambda \geq \lambda_{0}, 0 \leq t \leq T_{0}$. Here $C_{2}^{\prime \prime \prime}(p)$ is a nondecreasing function of $p \geq 0$.

To evaluate $\left(\langle D\rangle^{2} v, A_{0}(U+w)\langle D\rangle^{2}\left(A_{0}(U+w)^{-1} F(x, t, \lambda)\right)\right)$, we apply (3.16) to $A_{0}(U+w)^{-1}$ and use the following

Lemma 3.3. Let $f(x, \lambda) \in H^{\sigma}\left(\mathbf{R}^{n}\right), \sigma>n / 2$. Suppose there are constants $L_{\sigma}$ $L^{\prime}$ such that

$$
\|f(\cdot, \lambda)\|_{\sigma} \leq L_{\sigma} \lambda^{-\sigma-\theta}, \quad\|f(\cdot, \lambda)\|_{(\infty)} \leq L^{\prime} \lambda^{-\theta}
$$


for $\lambda \geq \lambda_{0}$. Then for $u \in H^{s}\left(\mathbf{R}^{n}\right), 0 \leq s \leq \sigma$, the product $f u \in H^{s}\left(\mathbf{R}^{n}\right)$ and

$$
\|f u\|_{s} \leq L_{s} \lambda^{s-\theta}\|u\|_{s}
$$

holds for $\lambda \geq \lambda_{0}$.

In fact, if $u \in H^{\sigma}\left(\mathbf{R}^{n}\right)$, then $f u \in H^{\sigma}\left(\mathbf{R}^{n}\right)$ and

$$
\|f u\|_{\sigma} \leq C\|f\|_{\sigma}\|u\|_{\sigma} \leq C L_{\sigma} \lambda^{\sigma-\theta}\|u\|_{\sigma} .
$$

If $u \in H^{0}\left(\mathbf{R}^{n}\right)$, then $f u \in H^{0}\left(\mathbf{R}^{n}\right)$ and

$$
\|f u\|_{0} \leq C\|f\|_{(\infty)}\|u\|_{0} \leq L^{\prime} \lambda^{-\theta}\|u\|_{0}
$$

Then by the interpolation, (3.28) holds.

Thus, we have

$$
\begin{aligned}
& \left|\left(\langle D\rangle^{2} v, A_{0}(U+w)\langle D\rangle^{2}\left(A_{0}(U+w)^{-1} F(x, t, \lambda)\right)\right)\right| \\
& \quad \leq K\left(\|w\|_{3}\right)\|v\|_{2}\|F\|_{2} \leq \lambda^{-1} L K\left(\|w\|_{3}\right)\|v\|_{2} \\
& \quad \leq \frac{1}{4}\|v\|_{2}^{2}+K\left(\|w\|_{3}\right)^{2} L^{2} \lambda^{-2}
\end{aligned}
$$

for $\lambda \geq \lambda_{0}, 0 \leq t \leq T_{0}$, with a nondecreasing function $K(p)$ of $p \geq 0$. Using the logarithmic convexity of the Sobolev scale, in particular, $\lambda^{2}\|v\|_{1}^{2} \leq$ $\frac{1}{2}\left(\lambda^{4}\|v\|_{0}^{2}+\|v\|_{2}^{2}\right)$, and (3.25), (3.26), (3.27), (3.15), we have

$$
\begin{aligned}
\frac{d}{d t}\left(\langle D\rangle^{2} v, A_{0}(U+w)\langle D\rangle^{2} v\right) \leq & 2 K_{2}\left(N, N_{1}, \lambda\right)\left(\langle D\rangle^{2} v, A_{0}(U+w)\langle D\rangle^{2} v\right) \\
& +\left(K_{20}\left(N, N_{1}\right) M_{2}+K_{21}\left(N, N_{1}\right) L^{2}\right) \lambda^{-2}
\end{aligned}
$$

with

$$
2 K_{2}\left(N, N_{1}, \lambda\right)=C_{20}+C_{21}(N)\left(N+N_{1}\right) \lambda^{s^{\prime}-3}
$$

for a constant $C_{20}$ and a nondecreasing function $C_{21}(p)$ of $p \geq 0$. Here $s^{\prime}<3$. (3.24) is now immediate.

We still need the estimate of $\|v(\cdot, x, \lambda)\|_{3}$. Let

$$
\tilde{v}_{k}(x, t, \lambda)=\frac{\partial}{\partial x_{k}}\left(\langle D\rangle^{2} v(x, t, \lambda)\right), \quad k=1, \ldots, n .
$$

Then it is easy to see that each $\tilde{v}_{k}$ satisfies the equation

$$
L_{U, 2}(w) \tilde{v}_{k}=\widetilde{F}_{k}
$$

where

$$
\begin{aligned}
\widetilde{F}_{k}(x, t, \lambda)= & \frac{\partial}{\partial x_{k}} F_{2}(x, t, \lambda)-\frac{\partial}{\partial x_{k}}(B(U+w)+C(U, w))\langle D\rangle^{2} v \\
& +2 \sum_{j=1}^{n} \frac{\partial}{\partial x_{k}}\left(A_{0}(U+w) \nabla_{x}\left(\tilde{A}_{j}(U+w)\right)\right) \cdot \nabla_{x} \frac{\partial}{\partial x_{j}} v,
\end{aligned}
$$

$k=1, \ldots, n$. Now from these, we estimate the inner products $\left(\tilde{v}_{k}, \widetilde{F}_{k}\right)$, using (3.22) and related estimates by an analogous argument as in the proof of Lemma 3.2. We then obtain the following 
Lemma 3.4. Under the same assumptions as Lemma 3.1, there are positive functions $K_{3}(p, q, \lambda), K_{30}(p, q), K_{31}(p, q)$ of $p \geq 0, q \geq 0$ and a constant $C_{1}>0$ such that

$(3.31)$

$$
\|v(\cdot, x, \lambda)\|_{3} \leq C_{1} e^{K_{3}\left(N, N_{1}, \lambda\right) t}\left(M^{2}+\left(K_{30}\left(N, N_{1}\right) M^{2}+K_{31}\left(N, N_{1}\right) L^{2}\right) t\right)^{1 / 2}
$$

for $0 \leq t \leq T_{0}$ and $\lambda \geq \lambda_{0}$. Here $K_{3}(p, q, \lambda)$ is given in the form

$$
2 K_{3}(p, q, \lambda)=C_{30}+C_{31}(p)(p+q) \lambda^{s^{\prime \prime}-3}
$$

with a constant $C_{30}$, a nondecreasing function $C_{31}(p)$ of $p \geq 0$, and $s^{\prime \prime}<3$.

Equations (3.31), (3.24), and (3.15) together with an interpolation argument lead to the following summary, by redefining constants and functions (in case of necessity).

Corollary 3.5. Under the assumptions of Lemma 3.1, there are constants $C_{0}, C_{1}$ and functions $C_{2}(p), K_{0}(p, q), K_{1}(p, q)$ such that

$$
\begin{aligned}
& \|v(\cdot, x, \lambda)\|_{s} \\
& \quad \leq C_{0} e^{K\left(N, N_{1}, \lambda\right) t}\left(M^{2}+\left(K_{0}\left(N, N_{1}\right) M^{2}+K_{1}\left(N, N_{1}\right) L^{2}\right) t\right)^{1 / 2} \lambda^{s-3}
\end{aligned}
$$

for $\lambda \geq \lambda_{0}, 0 \leq t \leq T_{0}$. Here

$$
2 K\left(N, N_{1}, \lambda\right)=C_{1}+C_{2}(N)\left(N+N_{1}\right) \lambda^{-\rho}, \quad \rho>0 .
$$

Using (3.13), we also have the following

Corollary 3.6. There is a nondecreasing function $K_{3}(p)$ of $p \geq 0$ independent of $\lambda \geq \lambda_{0}, 0 \leq t \leq T_{0}$, such that

$$
\left\|\frac{\partial}{\partial t} v(\cdot, t, \lambda)\right\|_{s} \leq K_{3}\left(\|w(\cdot, t, \lambda)\|_{3}\right)\|v(\cdot, t, \lambda)\|_{s+1}
$$

when $0 \leq s \leq 2$.

Now choose $N$ and $N_{1}$ large enough, for instance,

$$
N=2 C_{0} M e^{2 C_{1} T_{0}}, \quad N_{1}=K_{3}(N) N .
$$

Subsequently choose $T_{1}$ so small and $\lambda_{1}$ so large that

$$
\left(K_{0}\left(N, N_{1}\right) M^{2}+K_{1}\left(N, N_{1}\right) L^{2}\right) T_{1} \leq 3 M^{2},
$$

and

$$
C_{2}(N)\left(N+N_{1}\right) \lambda_{1}^{-\rho} \leq C_{1} .
$$

Then together with (3.33), (3.34), and (3.35) we see that

$$
\|v(\cdot, t, \lambda)\|_{s} \leq N \lambda^{s-3}, \quad s=0,1,2,3,
$$

and

$$
\left\|\frac{\partial}{\partial t} v(\cdot, t, \lambda)\right\|_{s} \leq N_{1} \lambda^{s-2}, \quad s=0,1,2,
$$

for $0 \leq t \leq T_{1}$ and $\lambda \geq \lambda_{1}$. In other words, if we denote by $\mathscr{X}\left(N, N_{1}, T_{1}, \lambda_{1}\right)$ the set of smooth $m$-vector-valued functions $w(x, t, \lambda)$ such that (3.11) and (3.12) hold for $0 \leq t \leq T_{1}$ and $\lambda \geq \lambda_{0}$, then the solution $v(x, t, \lambda)$ of (3.13), (3.14) belongs to $\mathscr{\mathscr { X }}\left(N, N_{1}, T_{1}, \lambda_{1}\right)$ provided $w(x, t, \lambda) \in \mathscr{X}\left(N, N_{1}, T_{1}, \lambda_{1}\right)$. 
In particular, all the $w^{k}(x, t, \lambda), k=1,2, \ldots$, defined successively from (3.10), belong to $\mathscr{X}\left(N, N_{1}, T_{1}, \lambda_{1}\right)$.

It is now convenient to introduce the norms

$$
\|w\|_{s, 3}=\sup _{0 \leq t \leq T_{1}, \lambda \geq \lambda_{1}} \lambda^{3-s}\|w(\cdot, t, \lambda)\|_{s},
$$

$0 \leq s \leq 3$, and

$$
\|w\|_{s, 2}=\sup _{0 \leq t \leq T_{1}, \lambda \geq \lambda_{1}} \lambda^{2-s}\|w(\cdot, t, \lambda)\|_{s}
$$

$0 \leq s \leq 2$, for $m$-vector-valued functions $w(x, t, \lambda)$.

Lemma 3.7. Suppose $v^{0}(x, t, \lambda), v^{1}(x, t, \lambda), v^{2}(x, t, \lambda) \in \mathscr{X}\left(N, N_{1}, T_{1}, \lambda_{1}\right)$ are related by

$$
\begin{aligned}
L_{U}\left(v^{0}\right) v^{1}=F, & \left.v^{1}\right|_{t=0}=v_{0}, \\
L_{U}\left(v^{1}\right) v^{2}=F, & \left.v^{2}\right|_{t=0}=v_{0} .
\end{aligned}
$$

Then there is a constant $C$ depending on $N, N_{1}$ such that

$$
\left\|v^{1}-v^{2}\right\|_{0,3} \leq C^{-1}\left(e^{C T_{1}}-1\right)^{1 / 2}\left\|v^{0}-v^{1}\right\|_{0,3}
$$

holds.

In fact, let $V^{1}=v^{1}-v^{2}$ and $V^{0}=v^{0}-v^{1}$. From (3.40), (3.41) we immediately get

$$
L_{U}\left(v^{0}\right) V^{1}=D\left(U, v^{0}, v^{1}, \partial v^{2}\right) V^{0},
$$

where

$$
D\left(U, v^{0}, v^{1}, \partial v^{2}\right) V^{0}=-\sum_{j=0}^{n} \int_{0}^{1} A_{j}^{\prime}\left(U+(1-\theta) v^{1}+\theta v^{0}\right)\left[V^{0}\right] d \theta \frac{\partial v^{2}}{\partial x_{j}}
$$

By the routine energy estimates, we have

$$
\frac{d}{d t}\left(V^{1}, A_{0}\left(U+v^{0}\right) V^{1}\right) \leq C\left(V^{1}, A_{0}\left(U+v^{0}\right) V^{1}\right)+\gamma\left\|V^{0}\right\|_{0}^{2},
$$

where $C=C\left(N, N_{1}\right)$ and $\gamma$ that of (1.2). Then (3.42) follows immediately.

Redefine $T_{1}>0$ if necessary and suppose

$$
\delta=C^{-1}\left(e^{C T_{1}}-1\right)^{1 / 2}<1 .
$$

Then these $w^{k}(x, t, \lambda)$ converge in the norm $\|\cdot\|_{0,3}$. In particular,

$$
\left\|w^{k}-w^{k-1}\right\|_{0,3} \leq \delta^{k-1} N, \quad k=1,2, \ldots
$$

Let $0<s<3$. Then for $p, q=1,2, \ldots, p>q$,

$$
\begin{aligned}
& \left\|w^{p}(\cdot, t, \lambda)-w^{q-1}(\cdot, t, \lambda)\right\|_{s} \\
& \quad \leq \sum_{k=q}^{p}\left\|w^{k}(\cdot, t, \lambda)-w^{k-1}(\cdot, t, \lambda)\right\|_{s} \\
& \quad \leq \sum_{k=q}^{p}\left\|w^{k}(\cdot, t, \lambda)-w^{k-1}(\cdot, t, \lambda)\right\|_{0}^{1-s / 3}\left\|w^{k}(\cdot, t, \lambda)-w^{k-1}(\cdot, t, \lambda)\right\|_{3}^{s / 3} \\
& \quad \leq(2 N)^{s / 3} \lambda^{s-3} N^{1-s / 3} \sum_{k=q}^{p}\left(\delta^{1-s / 3}\right)^{k-1} .
\end{aligned}
$$


Therefore, $w^{k}(\cdot, t, \lambda)$ converge in the norm $\|\cdot\|_{s, 3}$. Then using equation (3.13), we see that $\partial_{t} w^{k}(x, t, \lambda)$ converge in the norm $\|\cdot\|_{0,2}$, whence they also converge in the norm $\|\cdot\|_{s, 2}, 0<s<2$.

On the other hand, $w^{k}(x, t, \lambda)$ and $\partial w^{k}(x, t, \lambda) / \partial t$ are uniformly bounded in $H^{3}\left(\mathbf{R}^{n}\right)$, and $H^{2}\left(\mathbf{R}^{n}\right)$, respectively, with respect to $\lambda \geq \lambda_{1}$ and $0 \leq t \leq T_{1}$. It follows that $w^{k}(x, t, \lambda)$ and $\partial w^{k}(x, t, \lambda) / \partial t$ themselves weakly converge in $H^{3}\left(\mathbf{R}^{n}\right)$ and in $H^{2}\left(\mathbf{R}^{n}\right)$, respectively, to some $v(x, t, \lambda)$ and $v^{\prime}(x, t, \lambda)$. Furthermore, $v(x, t, \lambda)$ and $v^{\prime}(x, t, \lambda)$ are then uniform limits with respect to $t, \lambda$ of $w^{k}(x, t, \lambda)$ and $\partial w^{k}(x, t, \lambda) / \partial t$ in $H^{s}\left(\mathbf{R}^{n}\right)$ and $H^{s-1}\left(\mathbf{R}^{n}\right), s<3$, respectively. Thus $v^{\prime}=\partial v / \partial t$. The existence part of Theorem 1.5 is proved by putting $u=U+v$.

The uniqueness part is a consequence of the following

Lemma 3.8. Let the operator $L(w)$ satisfy the hypotheses stated in $\S 1$. Let $u^{1}(x, t)$ and $u^{2}(x, t)$ be two $m$-vector-valued functions defined for $x \in \mathbf{R}^{n}$, $0 \leq t \leq T$. Suppose $u^{1}(x, t)$ and $u^{2}(x, t)$ are $H^{1}\left(\mathbf{R}^{n}\right)$-valued continuous functions of $t, 0 \leq t \leq T$, and $\partial u^{1}(x, t) / \partial t$ and $\partial u^{2}(x, t) / \partial t$ are $H^{0}\left(\mathbf{R}^{n}\right)$ valued continuous functions of $t, 0 \leq t \leq T$. If $u^{1}(x, t)$ and $u^{2}(x, t)$ satisfy the equations

$$
L\left(u^{1}\right) u^{1}=0, \quad L\left(u^{2}\right) u^{2}=0
$$

with the same initial data at $t=0$, and if $u^{1}(x, t), u^{2}(x, t)$ together with all their first derivatives are uniformly bounded for $x \in \mathbf{R}^{n}, 0 \leq t \leq T$, then

$$
u^{1}(x, t) \equiv u^{2}(x, t) .
$$

In fact, derive the equation satisfied by $u^{1}-u^{2}$, and then apply the routine energy estimate.

\section{REFERENCES}

1. Y. Choquet-Bruhat, Ondes asymptotiques et approchées pour des systèmes d'équations aux derivées partielles nonlinéaires, J. Math. Pures Appl. 48 (1969), 117-158.

2. I. Daubechies, Orthonormal basis of compactly supported wavelets, Comm. Pure Appl. Math. 41 (1988), 909-996.

3. J. K. Hunter and J. B. Keller, Weakly nonlinear high frequency waves, Comm. Pure Appl. Math. 36 (1983), 547-569.

4. J. K. Hunter, A. Majda, and R. Rosales, Resonantly interacting, weakly nonlinear hyperbolic waves. II: Several space variables, Stud. Appl. Math. 75 (1986), 187-226.

5. T. Kato, The Cauchy problem for quasi-linear symmetric hyperbolic systems, Arch. Rational Mech. Anal. 58 (1975), 181-205.

6. __ Quasi-linear equations of evolution with applications to partial differential equations, Lecture Notes in Math., vol. 448, Springer-Verlag, Berlin, Heidelberg, and New York, 1975, pp. 25-70.

7. S. Klainerman, Global existence for nonlinear wave equation, Comm. Pure Appl. Math. 33 (1980), 43-101.

8. P. Lax, Hyperbolic systems of conservation laws and the mathematical theory of shock waves, CBMS-NSF Regional Conf. Ser. in Appl. Math., vol. 13, SIAM, Philadelphia, Pa., 1973.

9. A. Majda, Compressible fluid flow and systems of conservation laws in several space variables, Springer-Verlag, New York and Berlin, 1984. 
10. __ Nonlinear geometric optics for hyperbolic systems of conservation laws, Oscillation Theory, Computation, and Methods of Compensated Compactness, IMA Vol. Math. Appl., vol. 2, Springer-Verlag, Berlin and New York, 1986, pp. 115-165.

11. A. Yoshikawa, Note on the Taylor expansion of smooth functions defined on Sobolev spaces, Tsukuba J. Math. 15 (1991), 145-149.

Department of Applied Science, Faculty of Engineering, Kyushu University 36, FUKUOKA 812, JAPAN

E-mail address: c74474a@kyu-cc.cc.kyushu-u.ac.jp 